\title{
Evaluaciones subnacionales de la cobertura de las estadísticas vitales. Estudios recientes en América Latina*
}

\section{Evaluations of the Life Statistics Coverage at a Subnational Level. Recent Studies in Latin America}

\section{Evacuações subnacionais da cobertura das estatísticas vitais. Estudos recentes na América Latina}

Fecha de recepción: 17 de abril de 2017. Fecha de aprobación: 26 de agosto de 2017.

Fecha de publicación: 27 de mayo de 2019

DOI: https://doi.org/10.11144/Javeriana.rgps18-36.esce

\author{
Bruno Sebastián Ribotta ${ }^{a}$ \\ Universidad Nacional de Córdoba (UNC), Argentina \\ ORCID: 0000-0003-1943-0513 \\ Luisa María Salazar Acosta \\ Universidad Nacional de Salta (UNS), Argentina \\ ORCID: 0000-0003-0740-3534 \\ Carola Leticia Bertone \\ Centro de Investigación e Innovación Tecnológica (CENIIT) \\ Universidad Nacional de La Rioja, Argentina \\ ORCID: 0000-0001-9022-0688
}

Cómo citar este artículo: Ribotta BS, Salazar Acosta LM, Bertone CL. Evaluaciones subnacionales de la cobertura de las estadísticas vitales. Estudios recientes en América. Revista Gerencia y Políticas de Salud. 2019;18(36). https://doi.org/10.11144/Javeriana.rgps18-36.esce

\footnotetext{
Artículo de investigación

Los avances de esta investigación fueron presentados en la mesa redonda "Perspectivas de la comunidad académica sobre la evaluación de las estadísticas vitales y el registro civil," organizada por la División de Estadísticas de Naciones Unidas, en el marco del VII Congreso de la Asociación Latinoamericana de Población y XX Encontro Nacional de Estudos Populacionais (Foz do Iguazú, 17-22 de octubre de 2016), y en el panel "Cobertura de Estadísticas Vitales a nivel local,' de la VIII Reunión de la Red Latinoamericana y del Caribe para el Fortalecimiento de los Sistemas de Información en Salud, organizado por la Organización Panamericana de la Salud (Relacsis, Managua, 23-25 de mayo de 2017).

a_Autor de correspondencia. Correo electrónico: brunoribo@yahoo.com.ar
} 


\section{Resumen}

El logro de una buena cobertura de las estadísticas vitales (especialmente de los nacimientos y las defunciones) aún constituye un desafío muy importante en los países de América Latina. De ello depende el estudio de numerosas temáticas relacionadas con la salud de la población, como la situación y evolución de la natalidad, la fecundidad y la mortalidad. Al hacer un seguimiento de los avances en este aspecto, desde la demografía se ha notado que las técnicas de estimación más corrientes no funcionan adecuadamente al ser utilizadas en unidades geográficas o político-administrativas subnacionales. Para enfrentar el inconveniente, se están aplicando procedimientos poco frecuentes de estimación -o incluso, desarrollando otras técnicas- que permiten avanzar en el estudio de la cobertura de nacimientos y defunciones en diferentes niveles geográficos. En este trabajo se propone una revisión bibliográfica sobre las evaluaciones realizadas en América Latina desde el año 2000. Se recorta el análisis a estudios promovidos en el ámbito de los institutos nacionales de estadística y de los ministerios de salud, y considerando procedimientos principalmente de tipo directo.

Palabras clave: registro de estadísticas vitales, técnicas de investigación, recolección de datos, certificado de nacimiento, certificado de defunción, técnicas de estimación

\section{Abstract}

Attaining a good coverage of life statistics (especially births and deaths) still remains as a challenge in the Latin-American countries. The study of many topics related to the Latin-American population health such as the situation and evolution of the birth rates, fertility and mortality depends on attaining such objective. A follow-up to the progress on this concern under a demographic approach indicates that the ordinary estimation techniques do not prove to be suitable in geographic or jurisdictional units of a subnational level. To cope with this issue, some non-frequently used estimation procedures are now applied -indeed, new techniques are being developed-in order to make progress in the study of the birth and death recording coverage at different geographic levels. This work proposes a bibliographic review of the evaluations carried out in Latin America since 2000 to date. The analysis is narrowed to studies conducted by the national statistical institutes and the national ministries/departments of health and takes into account primarily procedures involving direct contact.

Keywords: vital statistics recording, research techniques, data collection, birth certificate, death certificate, estimation techniques

\section{Resumo}

Conseguir boa cobertura das estatísticas vitais (especialmente dos nascimentos e óbitos) ainda constitui desafio muito importante nos países da América Latina. De isso depende o estudo de numerosas temáticas relacionadas com a saúde da população, como a situação e evolução da natalidade, fecundidade e mortalidade. Ao monitorar os avanços neste aspecto, desde a demografia tem se notado que as técnicas de estimativa mais correntes não funcionam adequadamente quando utilizadas em unidades geográficas ou político-administrativas subnacionais. Para enfrentar o problema estão se aplicando procedimentos pouco frequentes de estimativa —ou mesmo, desenvolvendo outras técnicas-que permitem avançar no estudo da cobertura de nascimentos e óbitos em diferentes níveis geográficos. No trabalho propõe-se uma revisão bibliográfica sobre as avaliações realizadas na América Latina desde o 2000. A análise é reduzida a estudos promovidos no âmbito dos institutos nacionais de estatística e os ministérios de saúde, e considerando procedimentos principalmente de tipo direito.

Palavras chave: registro de estatísticas vitais, técnicas de pesquisa, coleta de dados, certidão de nascimento, atestado de óbito, técnicas de estimativa 


\section{Introducción}

Los sistemas de registro civil y estadísticas vitales que funcionan correctamente, proporcionan a los países información confiable y actualizada sobre el número y las características de los nacimientos y muertes de sus poblaciones, lo que permite definir programas de salud y desarrollo social más eficazmente (1). La importancia de dicha información puede ser constatada a propósito del seguimiento de los Objetivos de Desarrollo del Milenio (ODM), y adquiere nuevo interés en el marco de los desafíos que los países deberán afrontar como parte de la Agenda de Desarrollo post-2015, mediante los Objetivos de Desarrollo Sostenible (ODS). En este aspecto, las fuentes de datos alternativas como encuestas, censos o sistemas de vigilancia no son sustitutos adecuados desde el punto de vista estadístico y no proporcionan a los individuos la documentación legal que necesitan para beneficiarse de los servicios y participar plenamente en una sociedad moderna (2).

Para Naciones Unidas $(3,4)$ la calidad de la información sobre nacimientos y defunciones provista por los sistemas de registro civil y estadísticas vitales depende del cumplimiento de cuatro requisitos básicos: cobertura (que todos los hechos sean registrados), exactitud (que las características de estos sean inscriptas de manera precisa), oportunidad (que los resultados estén disponibles cuando se los necesita, en el menor tiempo posible) y disponibilidad (difusión en diferentes formatos, accesibles al usuario). Debido a la dificultad que representa garantizar estos requisitos, se han realizado esfuerzos importantes a nivel nacional e internacional para mejorar los sistemas de información correspondientes. En América Latina, un ejemplo destacado lo constituye el compromiso asumido por los Estados en las conferencias impulsadas por el Fondo para la Infancia de las Naciones Unidas (Unicef) para el registro civil de niños y niñas ${ }^{1}$. A su vez, y con relación a las estadísticas vitales, en el año 2008 la Organización Panamericana de la Salud (OPS) desarrolla una estrategia y un Plan de Acción Regional para el Fortalecimiento de las Estadísticas Vitales y de Salud (PEVS) ${ }^{2}$, que establece metas precisas basadas en la definición en el año 2005 de una línea de base para la cobertura de nacimientos y defunciones de 25 países de la región, y cuya aplicación está muy avanzada (5). En este marco se fundó la Red Latinoamericana y del Caribe para el Fortalecimiento de los Sistemas de Información de Salud (Relacsis)³.

Como corolario de estos esfuerzos, un avance destacado es el mejoramiento de la cobertura en el registro de los hechos vitales. Como recalca el Centro 
Latinoamericano y Caribeño de Demografía (Celade) (6), entre los años 1950-1955 y 2000-2005 a nivel regional se observan cambios relevantes tanto en los nacimientos (subregistro de $74 \%$ a $16 \%$ ) como en las defunciones (subregistro de $75 \%$ a $22 \%)^{4}$. A su vez, para el año 2013 la OPS muestra importantes avances en las metas de cobertura de los países que adhieren al PEVS; más específicamente, 18 de 25 países mantienen o mejoran la cobertura del registro de nacimientos, mientras 12 de 25 países accedieron al mismo logro en cuanto al registro de defunciones (7).

No obstante el progreso en los países de América Latina, resulta evidente la existencia de diferenciales "internos" en el registro de los hechos vitales (8). En este sentido, los promedios nacionales pueden ocultar realidades muy disímiles, que actúen negativamente en la construcción y el análisis de los indicadores de salud y, por lo tanto, en el alcance y la eficiencia en las políticas públicas que se propongan a partir de ellos. También se observa la limitación que representan las metodologías más corrientes para el estudio de la cobertura de nacimientos y defunciones en unidades político-administrativas menores (9). Por lo general, al tratarse de técnicas indirectas ${ }^{5}$, dos motivos impiden la utilización para evaluar la cobertura a nivel subnacional: el incumplimiento de los supuestos en que se basan (constancia o regularidad en el comportamiento de los componentes de la dinámica demográfica, ausencia de migración interna o internacional, etc.), y/o la imposibilidad de obtener resultados consistentes debido al escaso tamaño poblacional de algunas divisiones político-administrativas ${ }^{6}$.

Para superar limitaciones en la estimación de la cobertura de hechos vitales a nivel subnacional, los países de América Latina recurren cada vez más a metodologías directas, que impulsan desde sus ministerios de salud y/o institutos nacionales de estadísticas (INE) ${ }^{7}$. En los métodos directos se evalúa la cobertura de los nacimientos y las defunciones a través de la comparación con otros registros que contienen total o parcialmente la misma información, y provienen de una fuente independiente (por ejemplo, actas del registro civil, registros administrativos y sociales, listas provenientes de censos de población y encuestas, y sistemas de registro doble/paralelo). Adicionalmente, estas metodologías pueden implicar métodos de verificación basados en una "salida a terreno", como entrevistas y encuestas, incluyendo una modalidad especial para el estudio de las defunciones como las autopsias verbales. En cierta medida, los métodos directos de evaluación apuntan al origen del dato para realizar la evaluación, lo reconstruyen a partir de documentación emparentada de antemano o mediante el trabajo de campo. 
Las alternativas metodológicas directas para evaluar la cobertura de nacimientos y defunciones poseen mayor precisión en sus resultados que los métodos indirectos, y pueden aplicarse exitosamente en niveles subnacionales. Sin embargo, el uso de estos procedimientos representa una importante dificultad en términos técnicos y operativos, así como un elevado costo y demanda de tiempo ${ }^{8}$. Generalmente, estas limitaciones hacen que las metodologías directas para la evaluación de la cobertura de hechos vitales sean de aplicación poco frecuente, y por eso mismo, que resulten menos conocidas que los métodos indirectos. Ello también complica la acumulación del conocimiento y de la experiencia necesaria para adaptar los procedimientos existentes, o para crear nuevos, que puedan emplearse en países de pocos recursos y grandes urgencias de información.

En este trabajo se propone una primera aproximación al estudio de las evaluaciones de la cobertura de nacimientos y defunciones a nivel subnacional, que fueron realizadas desde el año 2000 en países de América Latina. Se propone para tal fin, una revisión del conjunto de publicaciones que hacen referencia a las evaluaciones mencionadas, para resumir sus principales características y los aspectos comunes. Se recorta la recopilación y el análisis a las evaluaciones promovidas en el ámbito de los institutos nacionales de estadística y/o de los ministerios de salud, considerando aquellas cuya principal estrategia metodológica sea direct ${ }^{9}$. Se entiende que el resumen de las características comunes más relevantes de las evaluaciones realizadas puede contribuir a orientar la mirada de los usuarios y productores de la información hacia los aspectos que merecen ser destacados, o que necesiten ser mejorados. Al mismo tiempo, la identificación de regularidades en las distintas evaluaciones realizadas también sería útil en la búsqueda de un estándar metodológico, de interés regional, que permita replicar las experiencias en otros países de la región, a la vez que podría ser usado en un mismo país, a lo largo del tiempo y en sus distintas jurisdicciones.

\section{Material y métodos}

Se realiza una búsqueda y selección de publicaciones (artículos, capítulos de libros o libros), referidos a prácticas de evaluación directa de la cobertura de nacimientos y defunciones a nivel subnacional. Los criterios de inclusión se relacionan con aspectos básicos que faciliten una caracterización preliminar de estas experiencias: la institución de pertenencia del autor del estudio o responsable de la publicación, el tipo de registro analizado, el nivel político administrativo al que se refiere la evaluación, y el tipo de metodología utilizada. 
En este sentido, se buscan y analizan textos que presenten experiencias oficiales de evaluación del registro de nacimientos y defunciones, que hayan considerado divisiones político-administrativas subnacionales (sean mayores o menores, como provincias, departamentos, municipios, etc.), y recurran a metodologías predominantemente directas $^{10}$. Los límites temporales de la revisión se extienden a publicaciones realizadas desde comienzos del presente siglo ${ }^{11}$.

En la figura 1 se observan tanto los insumos y fuentes de datos consideradas como los criterios de inclusión y las categorías analizadas.

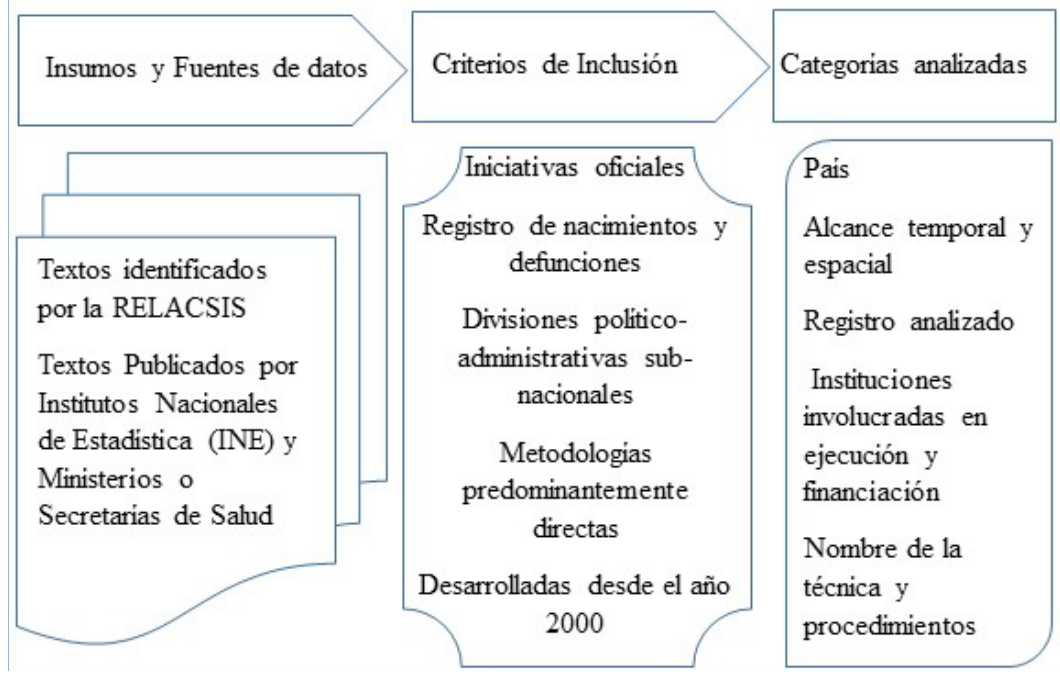

Figura 1. Aspectos metodológicos principales para la sistematización propuesta de las experiencias de evaluación de la cobertura de nacimientos y defunciones a nivel subnacional. América Latina. 2000-2015

Fuente: elaboración propia

La revisión bibliográfica se realizó teniendo en cuenta dos insumos. El primero corresponde a un listado de textos identificados por la Relacsis, que han sido analizados especialmente en el marco de un seminario online y un foro de discusión específico, organizados por el Grupo de Trabajo sobre Cobertura y Calidad de los sistemas de información en salud ${ }^{12}$. La segunda fuente corresponde a las páginas web de los institutos nacionales de estadística (INE) y de las direcciones de estadísticas de los ministerios o secretarías de salud de cada país, según los enlaces proporcionados por el Celade, División de Población de la Cepal ${ }^{13}$ y por la Relacsis de la OPS/OMS ${ }^{14}$, respectivamente. Tanto Celade como Relacsis son organismos conformados por investigadores y profesionales involucrados en 
la producción de datos y en el estudio de aspectos demográficos y de salud de Latinoamérica y el Caribe, con lo cual se considera pertinente tomar aquellas publicaciones que dichos organismos hayan recopilado.

Los documentos seleccionados en un primer momento fueron 29, aunque 6 de ellos se separaron de la lista dado que no cumplían algún criterio de inclusión ${ }^{15}$. Se procedió a la lectura y resumen de los textos, según las siguientes categorías: país, alcance temporal y espacial (año de las evaluaciones - unidades geográficas en que se desagrega el estudio), registro analizado (hecho vital —nacimiento y/o defunción-), instituciones involucradas en ejecución y financiación) y metodología (nombre de la técnica, procedimientos seguidos incluyendo muestreo si corresponde, principales resultados).

Se realizó un ordenamiento de los textos de acuerdo con las categorías antes mencionadas, de manera tal que la categoría país permite visualizar los estudios llevados a cabo en cada uno de ellos (véase tabla del anexo).

En la tabla 1, que se encuentra a continuación, se presentan los documentos seleccionados para llevar a cabo la revisión bibliográfica sobre evaluación de la cobertura de los registros vitales a nivel subnacional, con énfasis en las técnicas directas. Como puede apreciarse, los veintitrés documentos corresponden a cuatro países de la región: Argentina, Brasil, Colombia y Perú.

Tabla 1. Documentos seleccionados para la revisión bibliográfica sobre evaluación directa de la cobertura de los registros vitales en América Latina según categorías seleccionadas. Año 2000 en adelante

\begin{tabular}{|c|c|c|c|c|c|c|}
\hline \multirow[b]{2}{*}{ N. ${ }^{\circ}$} & \multirow[b]{2}{*}{ Texto } & \multirow[b]{2}{*}{$\frac{n}{\pi}$} & \multicolumn{2}{|r|}{ Alcance } & \multirow[b]{2}{*}{$\begin{array}{l}\text { Registro } \\
\text { evaluado }\end{array}$} & \multirow{2}{*}{ 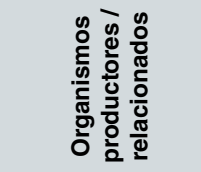 } \\
\hline & & & 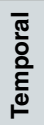 & $\begin{array}{l}\frac{\pi}{0} \\
\frac{\pi}{0} \\
\frac{0}{4} \\
\text { w }\end{array}$ & & \\
\hline 1 & $\begin{array}{l}\text { Fernández, Guevel, } \\
\text { Krupitzki, Marconi } \\
\text { y Massa (18) }\end{array}$ & 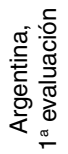 & ஜి & DAM & $\begin{array}{l}\text { Nacimientos -defun- } \\
\text { ciones infantiles }\end{array}$ & $\begin{array}{l}\text { DEIS, } \\
\text { OPS/OMS }\end{array}$ \\
\hline 2 & $\begin{array}{l}\text { Marconi, Fernández } \\
\text { y Guevel (19) }\end{array}$ & 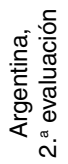 & 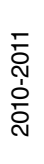 & $\begin{array}{l}\text { DAME } \\
\text { Aglomerados } \\
\text { urbanos }\end{array}$ & $\begin{array}{l}\text { Nacidos vivos-de- } \\
\text { funciones infantiles }\end{array}$ & $\begin{array}{l}\text { DEIS, } \\
\text { Unicef }\end{array}$ \\
\hline 4 & Szwarcwald et al. (21) & :응 & & DAM & & MINSAL, \\
\hline 5 & Frias et al. 22) & 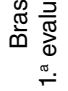 & ¿ & DAME & $\begin{array}{l}\text { funciones fetales e } \\
\text { infantiles }\end{array}$ & $\begin{array}{l}\text { Fundación Oswal- } \\
\text { do Fio Cruz }\end{array}$ \\
\hline
\end{tabular}




\begin{tabular}{|c|c|c|c|c|c|c|}
\hline \multirow[b]{2}{*}{ N. ${ }^{\circ}$} & \multirow[b]{2}{*}{ Texto } & \multirow[b]{2}{*}{$\frac{\mathscr{\omega}}{\pi}$} & \multicolumn{2}{|r|}{ Alcance } & \multirow[b]{2}{*}{$\begin{array}{l}\text { Registro } \\
\text { evaluado }\end{array}$} & \multirow{2}{*}{ 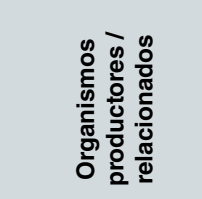 } \\
\hline & & & 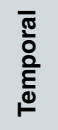 & $\begin{array}{l}\frac{\pi}{0} \\
\frac{\pi}{0} \\
\frac{\pi}{4}\end{array}$ & & \\
\hline 6 & Szwarcwald et al. (23) & \multirow{13}{*}{ 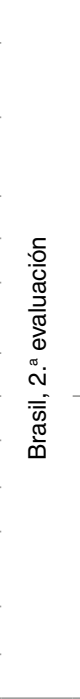 } & \multirow{13}{*}{$\stackrel{\infty}{\circ}$} & \multirow{7}{*}{$\begin{array}{l}\text { DAM } \\
\text { DAME }\end{array}$} & \multirow{13}{*}{$\begin{array}{l}\text { Nacimientos-de- } \\
\text { funciones totales e } \\
\text { infantiles }\end{array}$} & \multirow{13}{*}{$\begin{array}{l}\text { MINSAL, } \\
\text { Fundación Oswal } \\
\text { do Fio Cruz }\end{array}$} \\
\hline 7 & $\begin{array}{l}\text { Frias, Szwarcwald, de Souza, da } \\
\text { Silva de Almeida y Cabral Lira (24) }\end{array}$ & & & & & \\
\hline 8 & $\begin{array}{l}\text { Szwarcwald, de Frias, Júnior, } \\
\text { da Silva de Almeida y Neto (25) }\end{array}$ & & & & & \\
\hline 9 & Victora et al. (26) & & & & & \\
\hline 10 & Almeida y Szwarcwald (27) & & & & & \\
\hline 11 & $\begin{array}{l}\text { Szwarcwald, Escalante, Neto, } \\
\text { Junior y Victora (28) }\end{array}$ & & & & & \\
\hline 12 & França et al. (29) & & & & & \\
\hline 13 & Frias et al. (24) & & & & & \\
\hline 14 & Frias, Szwarcwald y Lira (30) & & & & & \\
\hline 15 & Szwarcwald et al. (31) & & & & & \\
\hline 16 & $\begin{array}{l}\text { Figueiroa, Vanderlei, Frias, } \\
\text { Carvalho y Szwarcwald (32) }\end{array}$ & & & & & \\
\hline 17 & Almeida y Szwarcwald (33) & & & & & \\
\hline 18 & Frias, Paulo Germano de et al. (34) & & & & & \\
\hline 19 & $\begin{array}{l}\text { Szwarcwald, Almeida } \\
\text { y Cortez-Escala nte (35) }\end{array}$ & \multirow{2}{*}{ 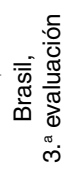 } & \multirow{2}{*}{$\stackrel{N}{\stackrel{N}{N}}$} & \multirow[t]{2}{*}{ DAM } & \multirow{2}{*}{$\begin{array}{l}\text { Total de } \\
\text { defunciones }\end{array}$} & \multirow{2}{*}{$\begin{array}{l}\text { Ministério da } \\
\text { Saúde, } \\
\text { Fundación Oswal- } \\
\text { do Fio Cruz }\end{array}$} \\
\hline 20 & Almeida, W.S. et al. (36) & & & & & \\
\hline 21 & $\begin{array}{l}\text { DANE y Dirección de Censos } \\
\text { y Demografía (37) }\end{array}$ & $\begin{array}{l}\frac{\pi}{0} \\
\frac{\pi}{E} \\
\frac{0}{0}\end{array}$ & ণิ & DAME & $\begin{array}{l}\text { Nacidos vivos-total } \\
\text { de las defunciones, } \\
\text { incluyendo algunas } \\
\text { relacionadas con } \\
\text { causas particulares }\end{array}$ & $\begin{array}{l}\text { DANE - Ministerio } \\
\text { de Protección } \\
\text { Social, UNFPA }\end{array}$ \\
\hline 22 & $\begin{array}{l}\text { Ávila Vargas Machuca, } \\
\text { Tavera Salazar } \\
\text { y Carrasco Gamarra (38) }\end{array}$ & \multirow[t]{2}{*}{$\frac{0}{2}$} & \multirow[t]{2}{*}{$\stackrel{m}{\grave{N}}$} & \multirow[t]{2}{*}{ DAME } & \multirow{2}{*}{$\begin{array}{l}\text { Defunciones } \\
\text { neonatales }\end{array}$} & $\begin{array}{l}\text { Ministerio de } \\
\text { Salud, }\end{array}$ \\
\hline 23 & Ávila, Tavera y Carrasco (39) & & & & & $\begin{array}{l}\text { UNICEF -OPS- } \\
\text { CARE }\end{array}$ \\
\hline
\end{tabular}

Nota: DAM: división administrativa mayor; DAME: división administrativa menor; DEIS: Dirección de Estadísticas e Información en Salud; OPS/OMS: Organización Panamericana de la Salud/Organización Mundial de la Salud; Unicef: Fondo de las Naciones Unidas para la Infancia; DANE: Departamento Administrativo Nacional de Estadística; UNFPA: Fondo de Población de las Naciones Unidas; CARE: Cooperative for Assistance and Relief Everywhere.

* La búsqueda activa se realiza para identificar hechos vitales del año 2000. Sin embargo, y como se describe en la tabla del anexo, de manera previa se realiza un análisis de la adecuación de las estadísticas vitales, basadas en una metodología indirecta que utiliza datos anteriores (1996-1998).

Fuente: elaboración propia 


\section{Resultados}

Como fuera anticipado, se dispuso de veintitrés documentos que destacan ocho evaluaciones de la cobertura de nacimientos y/o defunciones a nivel subnacional. A continuación, se sintetiza el resultado de la revisión bibliográfica, según las categorías que fueron seleccionadas para analizar el contenido de los textos. El detalle de lo trabajado se muestra en la tabla del anexo.

\section{Países}

Los textos seleccionados corresponden a evaluaciones principalmente directas de la cobertura de nacimientos y defunciones realizadas en cuatro países de la región: Argentina (tres evaluaciones en tres documentos), Brasil (tres evaluaciones en dieciocho documentos), Colombia (una evaluación en un documento) y Perú (una evaluación en dos documentos).

\section{Alcance temporal}

$\mathrm{Al}$ analizar la fecha de referencia de las evaluaciones (es decir, al año al que se refieren estas evaluaciones), se aprecia la siguiente distribución quinquenal:

- 2000-2004: Colombia (2002); primer estudio de Argentina (2003); primera investigación de Brasil (2000),

- 2005-2009: segundo análisis de Brasil (2008) y

- 2010-2015: segunda evaluación de Argentina (2010-2011); tercera de Argentina (2014-2015); tercer estudio de Brasil (2012); e investigación de Perú (2013).

Las fechas de referencia de las evaluaciones se ubican principalmente al inicio y final del periodo en estudio.

\section{Alcance espacial}

En cuanto a las unidades subnacionales de referencia para la evaluación de la cobertura de nacimientos y defunciones, en las prácticas escogidas se tiende a utilizar las siguientes estrategias: (1) la elección de la división político-administrativa más grande y (2) la selección de algunas unidades en particular, o de sectores específicos dentro de estas (como los municipios), a partir de procedimientos de muestreo intencional o probabilístico. 
En casi todas las evaluaciones seleccionadas, el estudio se realiza a nivel de la división administrativa mayor $(\mathrm{DAM})^{16}$. Las excepciones están representadas por la segunda evaluación de Argentina, que trabaja únicamente con grandes aglomerados urbanos, y con las prácticas de Brasil, que además de las DAM tiene en cuenta a los municipios, es decir, a la División Administrativa Menor del país (DAME).

Asimismo, en las evaluaciones seleccionadas se observa que el universo en estudio puede recortarse más allá de la división político-administrativa mencionada. Por ejemplo, en Argentina se elige intencionalmente a ciertas provincias (primer estudio) o aglomerados (segundo estudio), y dentro de estos a determinados establecimientos asistenciales (sector público, instituciones más concurridas, etc.). En Brasil, se analizan a las unidades federativas (UF) de determinadas regiones en particular (las que se incrementan en las distintas etapas del estudio) ${ }^{17}$, y dentro de las cuales se seleccionan determinados municipios.

En las evaluaciones mencionadas debe tenerse presente que las estrategias de segmentación del universo en estudio son estadísticamente diferentes. En las dos primeras prácticas de Argentina y la primera evaluación de Brasil la muestra utilizada es intencional. A diferencia de estos procedimientos, en los estudios de Brasil con referencia en los años 2008 y 2012 se utiliza una muestra probabilística de municipios de las regiones más perjudicadas por el problema. Esto posibilita posteriormente la generalización de los resultados a los municipios de otras regiones (véase anexo).

En Colombia se consideran todos los departamentos del país, pero mediante una muestra probabilística de los municipios que los integran. Finalmente, el único país que no recurre al muestreo es Perú. En este caso, debe tenerse presente que se considera solo a las muertes neonatales, es decir, un conjunto considerablemente más pequeño de casos.

\section{Registro evaluado}

En la mayoría de los estudios se considera tanto la cobertura de los nacimientos como de las defunciones. Sin embargo, el análisis se suele segmentar según diferentes criterios (por ejemplo, el total de defunciones según edad, o solo las muertes infantiles y sus componentes, etc.) Asimismo, en uno de los países la evaluación se aborda según el tipo de registro involucrado (legal o estadístico). 
En las evaluaciones de Argentina, Brasil y Colombia se ocupan de la cobertura de los nacimientos. A su vez, en todos los documentos seleccionados se tiene en cuenta la cobertura de las defunciones, aunque en algunos casos se analiza el universo total o una parte de este (por ejemplo, de acuerdo con una segmentación por edad o por la causa de las muertes). En la primera evaluación de Brasil se examinan las muertes fetales y de menores de un año ${ }^{18}$, en los estudios de Argentina de 2003 y 2010-2011 las muertes infanti$l e s^{19}$, y en el análisis de Perú las muertes neonatales. En la segunda y tercera experiencia realizada en Brasil, y en Colombia, se analiza a la totalidad de las defunciones; sin embargo, en el primer país se desagregan las muertes según dos grandes grupos de edad (2008 y 2012) ${ }^{20}$ e incluso según sexo (2012). En el segundo país se realiza además una indagación específica de algunos tipos de defunciones de algunas edades y/o causas de muerte en particular ${ }^{21}$.

Finalmente, es interesante mencionar que en el primer estudio de Argentina se distingue la evaluación de la "omisión legal" de los nacimientos y defunciones infantiles en tanto ausencia de la tramitación en el registro civil, de aquella que corresponde a la "subtransmisión" de los informes estadísticos desde dicha institución a las oficinas responsables de la elaboración de estadísticas. En este país los nacimientos y defunciones son registrados a través de un sistema de estadísticas vitales basado en el registro civil ${ }^{22}$.

\section{Instituciones responsables y/o colaboradoras}

Las instituciones responsables de llevar a cabo las evaluaciones seleccionadas pertenecen principalmente al ministerio de salud o sus dependencias, especialmente las dedicadas a la recolección, procesamiento y difusión de estadísticas de salud o el análisis epidemiológico. Excepcionalmente se observa la intervención de otros ministerios u organismos estatales (por ejemplo, en Colombia el estudio es llevado a cabo por la Departamento Administrativo Nacional de Estadística (DANE) y el Ministerio de Protección Social), y de ONG (Care-Perú). Solo en Brasil interviene una institución académica, la Fundación Oswaldo Cruz (Fiocruz), vinculada al Ministerio de la Salud.

Finalmente, se observa que tres países cuentan con el apoyo financiero de organismos internaciones (principalmente, OPS/OMS, Unicef y UNFPA) u ONG (Care-Perú). 


\section{Técnicas}

En los estudios elegidos, las técnicas directas que se utilizan para la evaluación de la cobertura (véase tabla del anexo) son:

1) Pareo de distintas fuentes de datos (Argentina, Colombia y Brasil): la técnica consiste en comparar el registro de interés con otros registros independientes que contengan total o parcialmente la misma información. A partir de la confrontación de materiales se elaboran listas bastante exhaustivas de hechos vitales, que identifican aquellos que no fueron incluidos en el sistema de registro (43, p. 134).

En las evaluaciones de Argentina se usan tres tipos distintos de fuentes de información para realizar el cotejo, a saber: registros hospitalarios (libros de partos, libros de defunciones, libros de morgue, historias clínicas, etc.), registro civil y de las personas (acta y libro de inscripción) y documentación estadística (informe estadístico de nacido vivo e informe estadístico de defunción).

En Colombia se emplean los mismos tipos de información, aunque en algunos casos bajo modalidades propias; las historias clínicas y los seriales de hechos vitales en instituciones de salud, registro civil, medicina legal y registros de inhumación. Como fuera anticipado, en la primera evaluación de Argentina la indagación se complementa con una encuesta a integrantes de los hogares que han estado relacionados con los hechos omitidos, y en Colombia, con autopsias verbales y consultas a parteras (solo aplicadas a determinados tipos de defunciones).

En Brasil se ha utilizado el Sistema Computarizado para el Control de la Muerte de la Compañía de Tecnología e Información de la Seguridad Social (Sisobi / Dataprev), empresa pública, que proporciona soluciones de tecnología de la información y comunicación para el perfeccionamiento y la ejecución de políticas sociales del Estado brasileño.

2) Captura-recaptura (Perú): como extensión de las técnicas de comparación directa, se desarrollan algunos procedimientos que consisten en emplear dos fuentes independientes para reunir información sobre los sucesos vitales. Básicamente, al cotejar ambas fuentes se determinan tres clases de hechos: los registrados en ambos sistemas (hechos concordantes), los registrados en la fuente 1 pero no en la 2 , y los registrados en la fuente 2 pero no en la 1 . A partir de ello, es posible estimar una cuarta clase de sucesos vitales: los no 
registrados por ninguno de los dos procedimientos. La suma de los cuatro tipos de hechos proporciona una estimación del número total de estos (43, p. 134) ${ }^{23}$.

Esta metodología se utiliza en Perú para evaluar el subregistro de mortalidad neonatal. En este país se compararon dos fuentes de información sobre mortalidad neonatal del año 2011, la base de datos del Subsistema de Vigilancia Epidemiológica Perinatal Neonatal (SNVEPN) de la Dirección General de Epidemiología del Ministerio de Salud y la base de defunciones del Sistema de Hechos Vitales (SHV) de la Oficina General de Estadística e Informática del Ministerio de Salud.

3) Búsqueda activa de nacimientos y defunciones (Brasil). Mediante esta técnica se intenta identificar los nacimientos o muertes que no han sido reportados a los sistemas de información sobre salud (SINASC y SIM, respectivamente), y/o las fallas en los procesos de certificación y notificación de tales hechos vitales. Se acude a terreno para recabar información de las siguientes fuentes: notarías, cementerios oficiales y no oficiales, establecimientos de salud, trabajadores de salud, informantes claves (parteras, líderes comunitarios, curanderos), funerarias y farmacias. Los datos se recogen en un instrumento estandarizado. Los hechos vitales que no figuran en el sistema son luego remitidos al Ministerio de Salud, lugar en donde se emiten y procesan los certificados de nacimiento o defunción que faltan $(45)^{24}$.

De manera complementaria al análisis basado en estos tres procedimientos de carácter directo, en algunos países se utilizan diferentes técnicas con "salida a campo" para profundizar el conocimiento de las causas del subregistro. Por ejemplo, las entrevistas y autopsias verbales realizadas en Colombia y las encuestas aplicadas en la primera evaluación de Argentina.

Como fuera anticipado, en algunos países también se utilizan de manera complementaria técnicas indirectas de análisis de la cobertura, tanto de manera previa como posterior a la aplicación de las técnicas directas. En el primer caso, se trata de los siguientes procedimientos:

- Comparación de la cantidad de nacimientos reportados por el sistema de estadísticas vitales (para un año determinado y/o incluyendo las inscripciones tardías), con los hijos nacidos vivos durante los doce meses anteriores reportados en un censo de población y vivienda. En la primera y tercera evaluación de Argentina, el cotejo de cifras permite estimar el porcentaje de subregistro de nacimientos. 
- Análisis de la adecuación de la información disponible sobre nacimientos y defunciones. En la primera evaluación de Brasil se realiza una apreciación crítica de cinco indicadores basados en estadísticas vitales (por ejemplo, la tasa bruta de mortalidad, la razón entre nacidos vivos registrados y estimados, el porcentaje de causas de muerte mal definidas, etc.), para cada uno de los municipios del país, lo que permite clasificarlos según la calidad de las informaciones del SIM y del SINASC. Entre otras consideraciones, el análisis de estos indicadores favorece la selección de los lugares en donde se efectúa la búsqueda activa.

Asimismo, y con posteridad a la utilización de métodos directos, también puede llevarse a cabo una comprobación de los resultados de la evaluación a partir de métodos indirectos. En la última evaluación de Brasil se utiliza una versión simplificada de la adecuación de la información de nacimientos y defunciones, para categorizar los municipios y facilitar la corrección de los datos, en lo que hace al modelaje estadístico y la estimación de factores de corrección.

Finalmente, en Colombia se analizan los resultados comparando el porcentaje de cobertura y las tasas de nacimiento y defunción, con estimaciones obtenidas de otras fuentes o con datos originales ${ }^{25}$. Por ejemplo, se contrastan los datos de registros con los presentes en una encuesta multipropósito que consulta sobre la existencia de partidas de nacimiento en niños menores de cinco años, y la proporcionalidad de la cobertura de nacimientos y defunciones, o dentro de estas, en las muertes perinatales respecto a las generales, entre otros procedimientos.

\section{Hallazgos seleccionados}

Las evaluaciones analizadas permiten identificar los niveles de cobertura a nivel nacional y subnacional, aunque con distinto alcance dependiendo del país. También posibilitan reconocer diferencias en el subregistro por edad y en determinadas causas de defunción, así como por diferenciales socioeconómicos (véase tabla del anexo).

En Brasil y Colombia proporcionan una estimación de la cobertura de los nacimientos y las defunciones, tanto en términos nacionales como subnacionales (incluyendo en el primer país al nivel municipal. Véase, por ejemplo, los resultados de la segunda evaluación en las figuras 2 y 3 ). 


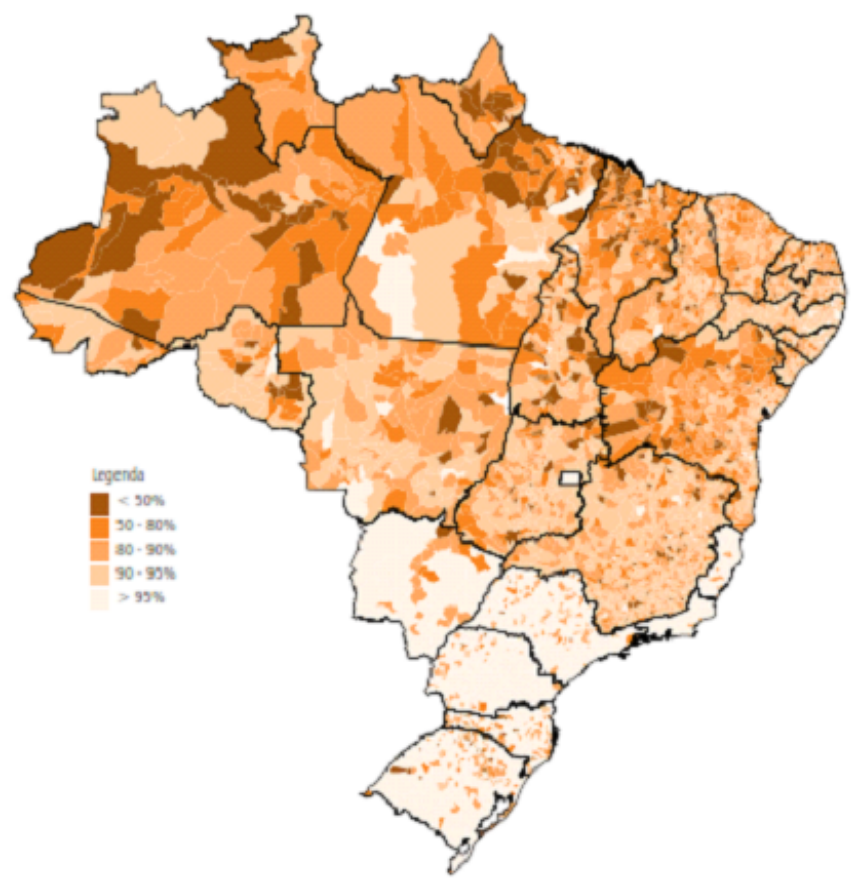

Figura 2. Brasil, distribución de los municipios según cobertura de las defunciones. 2008 Fuente: (23)

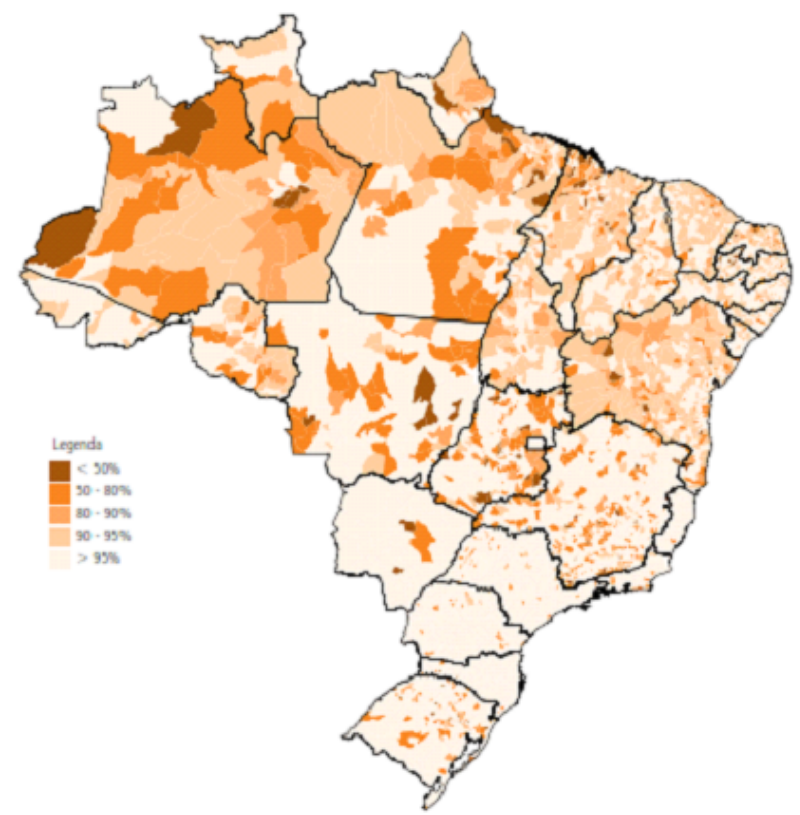

Figura 3. Brasil, distribución de los municipios según cobertura de los nacimientos. 2008

Fuente: (23) 
Dicha estimación también posee una desagregación geográfica similar en la evaluación peruana, pero para las defunciones neonatales exclusivamente. A diferencia de los resultados reportados por los estudios mencionados, en la primera evaluación de Argentina la estimación nacional de la cobertura resulta posible mediante un método indirecto, y únicamente para los nacimientos ${ }^{26}$. En este país, la cobertura de nacimientos y de defunciones infantiles reportada por el método directo concierne a los establecimientos de salud seleccionados de ciertas áreas de interés (provincias en la primera práctica y aglomerados urbanos en la segunda).

Es interesante notar que las estimaciones nacionales de cobertura obtenidas de manera directa (disponibles para Brasil y Colombia), arrojan en algunos casos un porcentaje de cobertura diferente al estimado por métodos indirectos. En Brasil, la evaluación correspondiente al año 2008 indica una cobertura de defunciones de $93 \%$ en ambos tipos de estimaciones. Sin embargo, en cuanto al registro de nacimientos se observa una diferencia que puede ser relevante (95\% según metodología directa y $91 \%$ en medición indirecta ${ }^{27}$.

En Colombia, la experiencia realizada en el año 2002 indica que eran registrados el $86 \%$ de los nacimientos y el $90 \%$ en defunciones. Otras mediciones, de carácter indirecto, destacan que la cobertura de nacimientos alcanzaba $84 \%$ y la de defunciones $82 \%$.

No obstante las diferencias de nivel observadas a propósito de las estimaciones nacionales de la cobertura de nacimientos y defunciones, las desagregaciones que posibilitan realizar las evaluaciones seleccionadas suman evidencias con relación a hipótesis que difícilmente pueden ser contrastadas. Así, se identifican notables contrastes geográficos en el registro de nacimientos y defunciones. Por ejemplo, en el año 2008 en Brasil la discrepancia entre las UF más y menos favorecidas económicamente, alcanza a veinticuatro puntos porcentuales para los nacimientos y diez puntos porcentuales para las defunciones ${ }^{29}$. En Colombia se aprecia una divergencia similar entre, por ejemplo, los departamentos de Bogotá y Chocó. Mientras que en el primero la cobertura de los nacimientos y defunciones asciende a $95 \%$, en el segundo es de $57 \%$ y $64 \%$, respectivamente.

Los estudios de Brasil y Colombia también confirman los diferenciales por edad y sexo en las defunciones, entre otras variables relevantes. 


\section{Conclusiones}

Si se consideran las dificultades relacionadas con la aplicación de métodos directos para la evaluación de la cobertura de estadísticas vitales, las prácticas analizadas muestran que están bien representadas en América Latina. Se trata de ocho evaluaciones encaradas en cuatro de los países más grandes de la región, que en conjunto incluyen al $53 \%$ de su población ${ }^{30}$. Argentina y Brasil cuentan con tres iniciativas cada uno. Colombia y Perú van por la primera. Se trata de una variedad de procedimientos de diferente complejidad técnica, operativa e incluso financiera, que han redundado en resultados relevantes, desde una determinación más fiel de la cobertura de los nacimientos y las defunciones a nivel nacional, a la consideración del registro en niveles locales, información que raramente está disponible. Entre otros resultados, contar con estas estimaciones locales permite contrastar hipótesis pocas veces exploradas sobre los diferenciales geográficos en el registro de hechos vitales, y una elaboración más exacta de ciertos indicadores, o su creación si antes no estaban disponibles. Esto último cobra especial relevancia en el marco del seguimiento de compromisos internos e internacionales de los países, como los ODS, que se plantean incluyendo a ese nivel político-administrativo.

A continuación se presentan algunas reflexiones que se deducen de la información analizada en este artículo.

\section{a). Extensión de las evaluaciones a otros países de la región}

Resulta fundamental continuar e incluso incrementar la difusión de las evaluaciones seleccionadas. En este sentido, no bastaría con insistir en la utilidad y el impacto de estos procedimientos, sino también en continuar y ampliar las iniciativas para la formación de los recursos humanos que las reproduzcan en sus países, mediante la producción y circulación de publicaciones específicas y la realización de talleres y seminarios de formación.

En este ámbito resulta interesante plantear un horizonte más específico de difusión, en la medida en que los manuales internacionales disponibles - sumamente útiles de por sí-, dan sin embargo cuenta de enfoques generales. Muchas de las evaluaciones seleccionadas involucran el tratamiento con éxito de problemáticas nacionales (por ejemplo, para la corrección de datos sobre la mortalidad infantil y materna), que podrían aplicarse en otros países, pero en el marco adecuado, que contemple sus especificidades. Por otro lado, los documentos elaborados en los países que han implementado estas 
iniciativas, son pocos o demasiados y responden a formatos diferentes. En este sentido, la difusión de las evaluaciones podría enriquecerse integrando contenidos particulares de estas, de manera uniformada. A ello nos referimos anteriormente en términos del establecimiento de un estándar, un conjunto de procedimientos que contemplen todos los aspectos necesarios para la evaluación de la cobertura de nacimientos y defunciones a nivel subnacional, pero adaptándose a las diferentes realidades de los países de América Latina.

\section{b). Ampliación de alcance temporal y espacial de las evaluaciones}

En el periodo analizado se observa una presencia continua de las iniciativas de evaluación directa de la cobertura de nacimientos y defunciones en América Latina. Sin embargo, al considerar por separado los países que las implementaron, se aprecia que las evaluaciones tienden a realizarse de manera errática en el tiempo, sin una temporalidad específica. Sin duda, esto remite a las condiciones que habrían facilitado la aplicación de estos métodos en la región, y de aquellas que las dificultan: el financiamiento, la disponibilidad de recursos humanos formados, etc.

En una primera lectura, la presencia de estas prácticas a pesar de las dificultades que podrían haber estado presentes, es congruente con el impulso otorgado por diferentes organismos nacionales e internacionales. En especial, se destaca el rol de algunas entidades que desarrollan planes de trabajo sistemáticos. Como fuera mencionado, en América Latina la OPS/ OMS viene trabajando desde hace más de una década con el PEVS, mientras otros organismos de Naciones Unidas cuentan con programas regulares de trabajo en esta materia (Celade, la División de Estadística de Naciones Unidas, Unicef, etc.) Quizá este sea un camino por seguir, la formalización de acuerdos que garanticen la continuidad de estas prácticas de evaluación en el tiempo.

\section{c). Implicancias de los diferentes sistemas de registro}

En la revisión realizada, se advierte escasa discusión sobre las implicancias teóricas y técnicas relacionadas con el tipo de registro evaluado (es decir, del componente legal o estadístico de estos). Lejos de representar una limitación de los enfoques metodológicos aplicados, la circunstancia podría vincularse con la coexistencia de diferentes sistemas de información sobre nacimientos y defunciones en América Latina, aspectos que no son tan evidentes para el lector externo. En este sentido, sería interesante actualizar los estados de situación sobre este particular, desde perspectivas comparativas ${ }^{31}$. 


\section{d). Instituciones involucradas}

En esta primera aproximación, no solo se ha observado la interrelación con organismos internacionales sino también con otros nacionales, principalmente del sector salud. Sin embargo, otros actores visualizados como los representantes del registro civil, y de las instituciones académicas, dedicadas a la investigación y la docencia podrían vincularse positivamente para la extensión de estas prácticas de evaluación. La contribución de los sectores académicos sería especialmente útil en problemáticas que surgen de la síntesis de los principales aspectos metodológicos de las evaluaciones, sobre todo con relación a una mayor contextualización conceptual y técnica de los métodos directos, y la necesidad de ampliar los procedimientos de consistencia de los resultados, cuestión que implica el uso de indicadores específicos y sobre todo de técnicas, en su mayoría indirectas, que representan cierta dificultad en términos estadístico-matemáticos o de procesamiento.

\section{e). Fortalecimiento de aspectos metodológicos}

Las distintas experiencias que se han realizado en la región podrían ser objeto de un análisis intensivo, que incluso las vincule con otras alternativas metodológicas en términos de evaluación y que resulte aplicable a diferentes países. Si bien las evaluaciones valoradas en este artículo habían sido realizadas en cuatro países, y ellos representan un sector mayoritario de la población de América Latina, sería útil dirigir la mirada a los 41 países restantes que, aun cuando más pequeños o igualmente diversos, también tienen necesidad de solucionar los problemas de calidad existentes en sus sistemas de información sobre nacimientos y defunciones.

El carácter naciente de estas evaluaciones y su diversidad entre los países que ya las están aplicando indicarían que los diferentes actores involucrados (institutos de estadística, registros civiles, ministerios de salud y la academia) deben ampliar los esfuerzos para integrar los distintos aportes y optimizar su aplicación. Al respecto, la contribución de los sectores académicos sería especialmente útil en problemáticas que surgen de la síntesis de los aspectos metodológicos. Entre ellos, los posibles aportes se articularían con:

- La posibilidad de una mayor contextualización conceptual y técnica de los métodos directos (mencionada con anterioridad a propósito de la producción de manuales específicos y planes de capacitación). 
- La necesidad de ampliar los procedimientos de consistencia de los resultados de las prácticas, cuestión que implica el uso de indicadores específicos y sobre todo de técnicas en su mayoría indirectas que representan cierta dificultad en términos estadístico-matemáticos o de procesamiento.

Finalmente, un aspecto central que merece ser llevado al centro de la discusión sobre la aplicabilidad de los métodos directos de evaluación de la cobertura, se refiere a la posibilidad de definir de un estándar regional.

Como fuera anticipado, en este artículo se propone una primera aproximación a la caracterización de las evaluaciones relevadas. Se espera complementar el estudio en el futuro, con la identificación y categorización de aspectos tales como las limitaciones que enfrenta la aplicación de los métodos (sea en términos teóricos y técnicos, operativos o financieros), los cambios que han propiciado estas iniciativas en la producción de datos o en cuestiones tales como la legislación relacionada con los hechos vitales, y los desafíos que quedan pendientes, entre otras temáticas.

\section{Referencias}

1. Mikkelsen L, Phillips DE, AbouZahr C, Setel PW, De Savigny D, Lozano R, et al. A global assessment of civil registration and vital statistics systems: monitoring data quality and progress. Lancet. 2015;386(10001):1395-406. doi: 10.1016/ S0140-6736(15)60171-4.

2. AbouZahr C, De Savigny D, Mikkelsen L, Setel PW, Lozano R, Lopez AD. Towards universal civil registration and vital statistics systems: The time is now. Lancet. 2015;386(10001):1407-18. doi: 10.1016/S0140-6736(15)60170-2.

3. Naciones Unidas. Departamento de Asuntos Económicos y Sociales. División de Estadística. Principios y recomendaciones para un sistema de estadísticas vitales. Revisión 2. Nueva York: NNUU; 2003.

4. Naciones Unidas. Departamento de Asuntos Económicos y Sociales. División de Estadística. Principios y recomendaciones para un sistema de estadísticas vitales. Revisión 3. New York: Naciones Unidas; 2014.

5. AbouZahr C, De Savigny D, Mikkelsen L, Setel PW, Lozano R, Nichols E, et al. Civil registration and vital statistics: Progress in the data revolution for counting and accountability. Lancet. 2015;386(10001):1373-85. doi: 10.1016/S01406736(15)60173-8. 
6. Celade. La calidad de las estadísticas vitales en la América Latina. En: II Reunión Regional sobre Evaluación y Estimaciones Demográficas con base en Información Censal. Santiago de Chile: Cepal/Celade; 2012.

7. Ruiz PL, Giusti A, Fernández G, Gerger A. Progresos en la cobertura de nacimientos y defunciones - Región de las Américas. En: VI Reunión de la Red Latinoamericana y del Caribe para el Fortalecimiento de los Sistemas de Información de Salud (RELACSIS). Bogotá: OPS/OMS, MSH/USAID, CIDA; 2014.

8. OPS/OMS. Situación de las estadísticas vitales, de morbilidad y de recursos y servicios en salud de los países de las Américas (informe regional). Washington D. C.: OPS/OMS; 2007.

9. Murray CJL, Rajaratnam JK, Marcus J, Laakso T, Lopez AD. What can we conclude from death registration? Improved methods for evaluating completeness. PLoS Med. Public Library of Science; 2010;7(4):e1000262. doi: 10.1371/journal. pmed.1000262.

10. De Lima EEC, Queiroz BL. Evolution of the deaths registry system in Brazil: associations with changes in the mortality profile, under-registration of death counts, and ill-defined causes of death. Cad Saude Publica. 2014;30(8):1721-30. doi: 10.1590/0102-311X00131113.

11. Fondo de las Naciones Unidas para la Infancia (Unicef) - México, Instituto Nacional de Estadística y Geografía (INEGI). Derecho a la identidad. La cobertura del registro de nacimiento en México en 1999 y 2009. México DF: Unicef - Inegi; 2012.

12. Moultrie T, Dorrington R, Hill A, Hill K, Timæus I, Zaba B. Tools for demographic estimation. Paris: IUSSP - UNFPA; 2013.

13. OMS. La salud y los objetivos de desarrollo del milenio. Ginebra: OMS; 2005.

14. Schmid B, Silva NN. Estimação de sub-registro de nascidos vivos pelo método de captura e recaptura, Sergipe. Rev Saúde Pública. 2011;45(6):1088-98. Disponible en: https://www.scielosp.org/pdf/rsp/2011.v45n6/1088-1098/en

15. Schmid B. Estimação de sub-registro de nascidos vivos pelo método de captura e recaptura. São Paulo: Universidade de São Paulo; 2009.

16. Chaparro Narváez PE. Calidad de la información de mortalidad en menores de un año en Bogotá durante 2005. Bogotá: Universidad Nacional de Colombia; 2013.

17. Pérez E, Meneses E. El registro de los nacimientos. Una revaloración de la fuente para medir la fecundidad en México. En: X Reunión Nacional de Investigación Demográfica en México Escenarios Demográficos y Política de Población en el Siglo XXI. México D. F.: Somede; 2010. p. 1-15.

18. Fernández M, Guevel C, Krupitzki H, Marconi E, Massa C. Omisión de registro de nacimientos y muertes infantiles Magnitud, desigualdades y causas. Buenos Aires: Organización Panamericana de la Salud - Ministerio de Salud; 2008. 
19. Marconi E, Fernández M, Guevel CG. El derecho al registro universal y oportuno de niñas y niños. Avances y desafíos para la normativa legal y las estadísticas vitales. Buenos Aires: Fondo de las Naciones Unidas para la Infancia (Unicef); 2013.

20. Bomben E, Calvelo L, Calva G, De Paula M, Fernández M, Lester N, Massa C, Minué N, Santoro A, Vidal L. Registro de nacimientos en Argentina. Un estudio sobre la cobertura legal y estadística. Buenos Aires: Presidencia de la Nación - Unicef; 2017.

21. Szwarcwald CL, Leal MDC, Andrade CLT, De Souza Jr. PRB. Estimação da mortalidade infantil no Brasil: o que dizem as informações sobre óbitos e nascimentos do Ministério da Saúde? Cad Saude Publica. 2002;18(6):1725-36. Disponible en: https://www.scielosp.org/pdf/csp/2002.v18n6/1725-1736/pt

22. Frias PG, Pereira PMH, Andrade CLT, Szwarcwald CL. Sistema de Informações sobre Mortalidade: estudo de caso em municípios com precariedade dos dados. Cad Saude Publica. 2008;24(10):2257-66. Disponible en: https://www.scielosp. org/pdf/csp/2008.v24n10/2257-2266/pt

23. Szwarcwald CL, Morais Neto OL, Frias PG, Souza Jr. PRB, Cortez-Escalante JJ, Lima R, et al. Busca ativa de óbitos e nascimentos no Nordeste e na Amazônia Legal: Estimação das coberturas do SIM e do Sinasc nos municípios brasileiros. En: Brasil, Ministério da Saúde, Secretaria de Vigilância em Saúde, Departamento de Análise de Situação em Saude, editores. Saúde Brasil 2010: uma análise da situação de saúde e de evidências selecionadas de impacto de ações de vigilância em saúde. Brasilia: Ministério da Saúde; 2011. p. 79-98.

24. Frias PG, Szwarcwald CL, Souza PRB, Da Silva de Almeida W, Cabral Lira PI. Correcting vital information: Estimating infant mortality, Brazil, 2000-2009. Rev Saude Publica. 2013;47(6):1048-58. doi: 10.1590/S0034-8910.2013047004839.

25. Szwarcwald CL, Morais Neto OL, Frias PG, Souza Jr. PRB, Cortez-Escalante JJ, Lima R, et al. Busca ativa de óbitos e nascimentos no Nordeste e na Amazônia Legal: Estimação da mortalidade infantil nos municípios brasileiros. En: Brasil, Ministério da Saúde, Secretaria de Vigilância em Saúde, Departamento de Análise de Situação em Saude. Saúde Brasil 2010: uma análise da situação de saúde e de evidências selecionadas de impacto de ações de vigilância em saúde. Brasília: Ministério da Saúde; 2011. p. 99-114.

26. Victora CG, Aquino EM, Do Carmo Leal M, Monteiro CA, Barros FC, Szwarcwald CL. Maternal and child health in Brazil: Progress and challenges. Lancet. 2011;377(9780):1863-76. doi: 10.1016/S0140-6736(11)60138-4.

27. Almeida WDS, Szwarcwald CL. Mortalidade infantil e acesso geográfico ao parto nos municípios brasileiros. Rev Saude Publica. 2012;46(1):68-76. Disponible en: https://www.scielosp.org/pdf/rsp/2012.v46n1/68-76/en

28. Szwarcwald CL, Escalante JJC, Neto LR, Junior PRB, Victora CG. Estimação da razão de mortalidade materna no Brasil, 2008-2011. Cad Saúde Pública, Rio Janeiro. 2014;30(Supl.1):S71-83. doi: 10.1590/0102-311X00125313. 
29. França E, Teixeira R, Ishitani L, Duncan BB, Cortez-Escalante JJ, Morais Neto OLD, et al. Ill-defined causes of death in Brazil: a redistribution method based on the investigation of such causes. Rev Saude Publica. 2014;48(4):671-81. doi: 10.1590/S0034-8910.2014048005146.

30. Frias PG, Szwarcwald CL, Lira PIC. Avaliação dos sistemas de informações sobre nascidos vivos e óbitos no Brasil na década de 2000. 2014;30(10):2068-80. doi: 10.1590/0102-311X00196113.

31. Szwarcwald C, de Frias P, Júnior PRB, Da Silva de Almeida W, Neto OL. Correction of vital statistics based on a proactive search of deaths and live births: evidence from a study of the North and Northeast regions of Brazil. Popul Health Metr. 2014;12(16):16. doi: 10.1186/1478-7954-12-16.

32. Figueiroa BDQ, Vanderlei LCDM, Frias PG, Carvalho PI, Szwarcwald CL. Analysis of coverage in the mortality information system in Olinda, Pernambuco State, Brazil. Cad Saude Publica. 2013;29(3):475-84. doi: 10.1590/S0102311X2013000300006.

33. Almeida W, Szwarcwald CL. Mortalidade infantil nos municípios brasileiros: uma proposta de método de estimação. Rev Bras Saúde Matern Infant. 2014;15(1):33142. doi: 10.1590/S1519-38292014000400003.

34. Frias PG, Szwarcwald CL, Morais Neto OL, Leal M, Cortez-Escalante JJ, Souza Junior PRB, et al. Utilização das informações vitais para a estimação de indicadores de mortalidade no Brasil: da busca ativa de eventos ao desenvolvimento de métodos. Cad Saude Publica. 2017;33(3):1-13. doi: 10.1590/0102-311X00206015.

35. Szwarcwald CL, Almeida W, Cortez-Escalante J. Foro Cobertura 2016 - Webinar 2 - Corrección de las estadísticas vitales en los municipios Brasileños: Búsqueda activa de nacimientos y defunciones. Brasil: Relacsis; 2016.

36. Almeida WS, Szwarcwald CL, Frias PG, Souza Júnior PRB, Lima RB, Rabello Neto DL, et al. Captação de óbitos não informados ao Ministério da Saúde: pesquisa de busca ativa de óbitos em municípios brasileiros. Rev Bras Epidemiol. 2017;20(2):200-11. doi: 10.1590/1980-5497201700020002.

37. Colombia, DANE, Dirección de Censos y Demografía. La mortalidad materna y perinatal en Colombia en los albores del siglo XXI. Estimación del subregistro de nacimientos y defunciones y estimaciones ajustadas de nacimientos, mortalidad materna y perinatal por departamentos. Bogotá: DANE, UNFPA, Ministerio de Protección Social; 2006.

38. Ávila Vargas Machuca J, Tavera Salazar M, Carrasco Gamarra M. Mortalidad neonatal en el Perú y sus departamentos 2011-2012. Lima: Ministerio de Salud - Dirección General de Epidemiología, Unicef, OPS/OMS, Care, Union Europea; 2013. 
39. Ávila J, Tavera M, Carrasco M. Características epidemiológicas de la mortalidad neonatal en el Perú, 2011-2012. Rev Peru Med Exp Salud Publica. 2015;32(3):42030. Disponible en: http://www.scielo.org.pe/scielo.php?script=sci_arttext\&pi$\mathrm{d}=\mathrm{S} 1726-46342015000300003 \& \operatorname{lng}=\mathrm{es} \& \mathrm{nrm}=\mathrm{iso}>$. ISSN 1726-4634

40. Brasil, Ministério da Saúde. A experiência brasileira em sistemas de informação em saúde. OPS/OMS, Fundacao Oswaldo Cruz, editores. Brasilia: Ministério da Saúde, Secretaria de Atenção à Saúde, Departamento de Atenção Especializada, Brasil; 2009.

41. Colombia, DANE, Dirección de Censos y Demografía. Metodología estadísticas vitales. Bogotá: DANE; 2012.

42. Perú, INEI. El sistema de registro civil y estadísticas vitales. En: El crecimiento natural y sus componentes : Nacimientos y defunciones 2009-2013 (visión departamental). Lima: INEI; 2015. p. 19-23.

43. OPS/OMS, Fondo de las Naciones Unidas para la Infancia (Unicef). Actualización del 2014. Eliminación de la transmisión maternoinfantil del VIH y la sífilis en las Américas. Washington: OPS/OMS; 2014.

44. Chandra Sekar C, Edwards Deming W. On a method of estimating birth and death rates and the extent of registration. Journal of the American statistical Association. 1949:44:101-15. doi: 10.1590/1980-5497201700020002.

45. Szwarcwald CL. Strategies for improving the monitoring of vital events in Brazil. Int J Epidemiol. 2008;37(4):738-44. doi: 10.1093/ije/dyn130.

46. Velázquez Lerna R. Registros administrativos sobre salud en México. México D. F.: Inegi; 2012.

47. Chackiel J. Evaluación post-empadronamiento de la cobertura en los censos de población. Notas Población. 2010;91:43-71. Disponible en: https://repositorio.cepal. org/bitstream/handle/11362/12872/np91043071_es.pdf?sequence=1\&isAllowed =y

48. United Nations, Department of Economic and Social Affairs PD. World population prospects: The 2015 revision, volume I: Comprehensive tables (ST/ESA/ SER.A/379). Nueva York: United Nations; 2015.

49. United Nations, Department of Economic and Social Affairs PD. World population prospects: The 2015 revision, key findings and advance tables. Working paper ESA/P/WP.241. Nueva York: United Nations; 2015.

50. Celade. Observatorio Demográfico 2015. Proyecciones de población. Santiago de Chile: Naciones Unidas; 2016. 


\section{Anexo}

\section{Países de América Latina. Síntesis de características seleccionadas en eva- luaciones de la cobertura del registro de nacimientos y defunciones. Periodo de referencia de la evaluación: años 2000-2015}

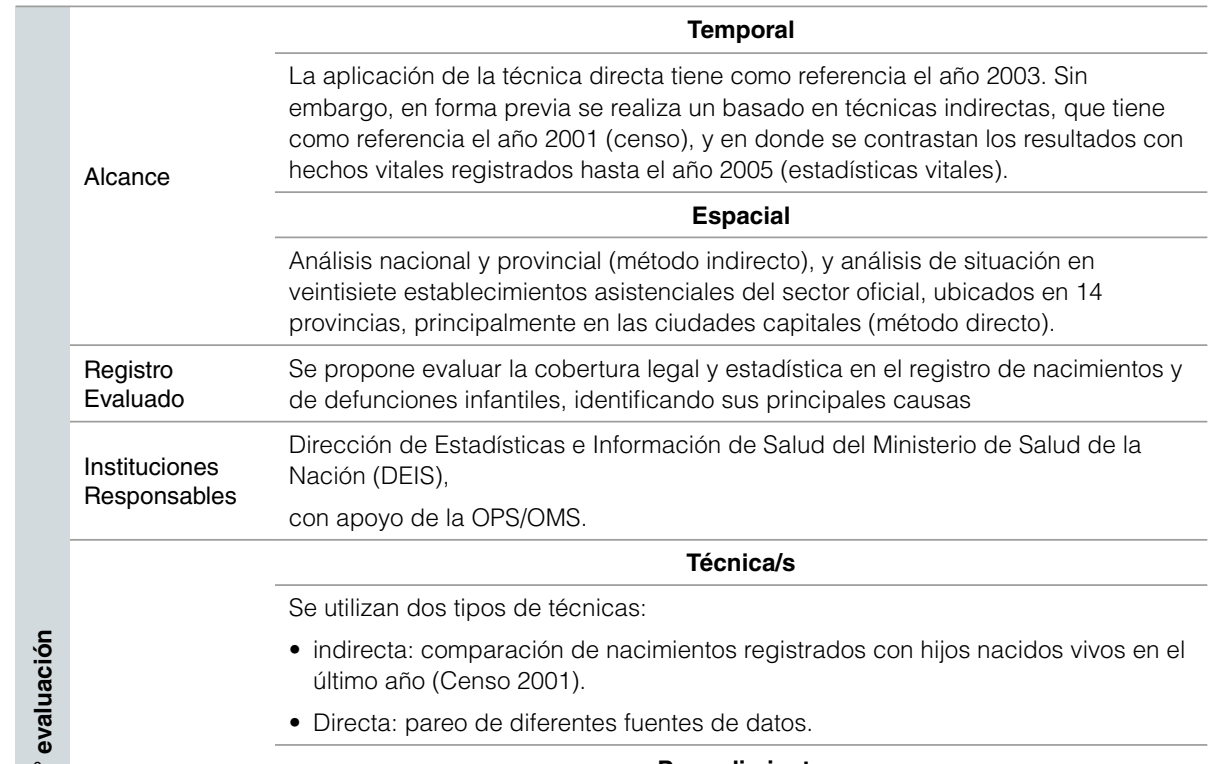

\section{Procedimiento}

Indirecto; se analizan resultados del Censo 2001 (hijos nacidos vivos), para el total del país y sus provincias. Estos datos se comparan con nacimientos registrados. Dicha aplicación no es posible para las defunciones infantiles.

Directo; Se realiza pareo de fuentes de datos o documentación complementaria: registros hospitalarios (libros de partos, libros de defunciones, libros de morgue, historias clínicas, etc.), registros civiles (acta y libro de inscripción en el Registro

Metodología Civil) y documentación estadística (Informe Estadístico de Nacido Vivo e Informe Estadístico de Defunción). Se pretende con ello identificar los sucesos vitales no anotados, mediante la consideración de listas "completas" de todos los nacimientos y defunciones infantiles bajo estudio.

Al respecto es posible distinguir dos variantes:

- Los registros hospitalarios y la información del Registro Civil permiten identificar casos de subregistro legal (no inscripción de los correspondientes hechos vitales en el Registro Civil).

- La información de los registros civiles y el Informe Estadístico de Nacido Vivo y el Informe Estadístico de Defunción, permiten cuantificar los casos de subtransmisión estadística de información (inscripto legalmente pero no contabilizado estadísticamente).

Para la identificación de las causas de omisión desde la perspectiva poblacional, se utiliza una encuesta aplicada a los hogares de los niños (vivos o fallecidos) que no fueron inscritos. 


\begin{tabular}{|c|c|c|}
\hline \multirow{6}{*}{ 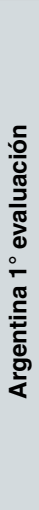 } & & Hallazgos Seleccionados \\
\hline & & $\begin{array}{l}\text { Al comparar con datos censales, se encuentra un } 6 \% \text { de omisión del registro de } \\
\text { nacimiento al cabo de un año de ocurrido y en } 3 \% \text { luego de cinco años. }\end{array}$ \\
\hline & & $\begin{array}{l}\text { Los resultados muestran además importantes diferencias entre jurisdicciones. } \\
\text { El estudio señaló también que las omisiones en el registro de nacimiento podían } \\
\text { estar acompañadas por omisiones de defunciones, con implicancias sobre las } \\
\text { tasas de natalidad y mortalidad infantil. }\end{array}$ \\
\hline & & $\begin{array}{l}\text { El pareo de fuentes permitió detectar elevados niveles de omisión de defunciones } \\
\text { infantiles en los establecimientos y áreas seleccionadas de las provincias de } \\
\text { Catamarca, Santiago del Estero, Tucumán y Misiones, así como en el interior de la } \\
\text { provincia de Formosa. }\end{array}$ \\
\hline & & La subtransmisión es mínima. \\
\hline & $\begin{array}{l}\text { Texto y Año de } \\
\text { Publicación }\end{array}$ & Fernández, Guevel, Krupitzki, Marconi y Massa (2008) (12) \\
\hline
\end{tabular}

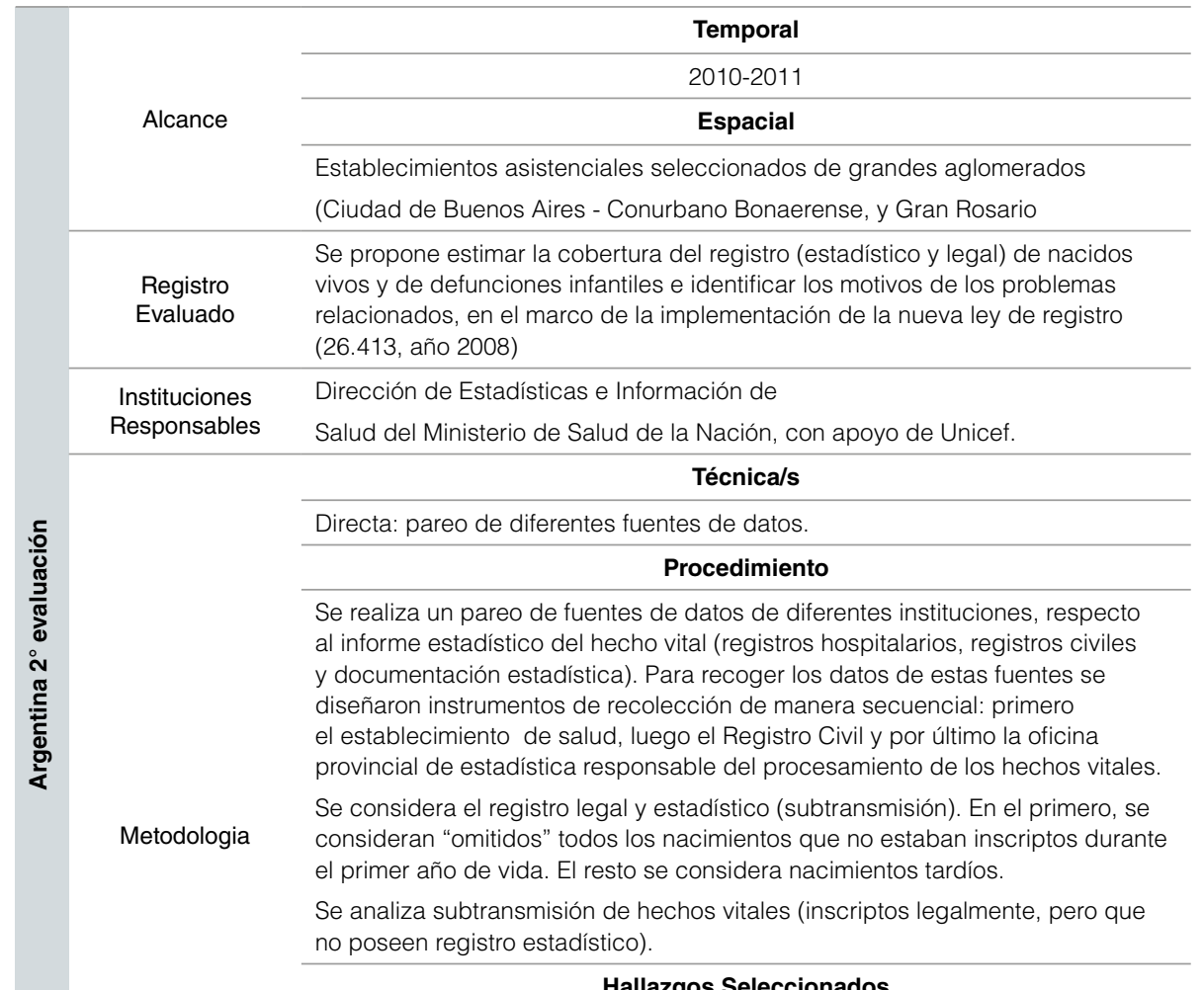

\section{Hallazgos Seleccionados}

En los establecimientos relevados en el estudio la cobertura de registro de nacimientos y defunciones infantiles es cercana al $100 \%$. No obstante, la calidad de la información es deficitaria, poniendo de manifiesto debilidades, en especial de los sistemas de registro administrativos, médicos y estadísticos que, a su vez, generan déficits en la información producida por el sistema de estadísticas vitales y los registros civiles.

Texto y Año de Marconi, Fernández, y Guevel, (2013) (13)

Publicación 


\begin{tabular}{|c|c|c|}
\hline \multirow{15}{*}{ 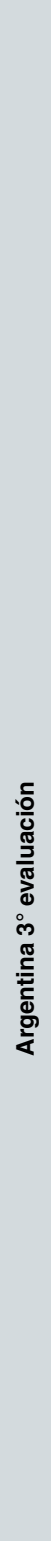 } & \multirow{5}{*}{ Alcance } & Temporal \\
\hline & & $\begin{array}{l}\text { Se trabajó con información de los años } 2014 \text { y } 2015 \text { y sólo se ha logrado } \\
\text { recuperar una parte, quizás la más importante de los nacimientos inscriptos } \\
\text { tardíamente, pero es probable que posteriormente se registren nacimientos } \\
\text { ocurridos también en } 2014 \text {. }\end{array}$ \\
\hline & & Espacial \\
\hline & & $\begin{array}{l}\text { Análisis nacional y provincial y análisis de situación en } 5 \text { establecimientos de } \\
\text { salud seleccionados ubicados en tres provincias: Buenos Aires, La Rioja; Santa } \\
\text { Fe. }\end{array}$ \\
\hline & & $\begin{array}{l}\text { Se priorizó en la selección de los establecimientos a aquéllos que concentran } \\
\text { una ampliaproporción de nacidos vivos en las áreas que están localizados y } \\
\text { son efectores de salud con altonivel de resolución }\end{array}$ \\
\hline & $\begin{array}{l}\text { Registro } \\
\text { Evaluado }\end{array}$ & $\begin{array}{l}\text { Se propone estimar la cobertura del registro legal y estadístico de nacidos } \\
\text { vivos hacia el año } 2015 \text { y comparar el Censo Nacional de Población, Hogares } \\
\text { y Viviendas } 2010 \text { del INDEC. Complementariamente, se analiza la información } \\
\text { sobre identificación de } 0 \text { años del Renaper. }\end{array}$ \\
\hline & $\begin{array}{l}\text { Instituciones } \\
\text { Responsables }\end{array}$ & $\begin{array}{l}\text { Dirección de Estadísticas e Información de Salud (DEIS) del Ministerio de } \\
\text { Salud de la Nación (MSAL) y el Fondo de las Naciones Unidas para la Infancia } \\
\text { (Unicef). }\end{array}$ \\
\hline & \multirow{8}{*}{ Metodologia } & Técnica/s \\
\hline & & $\begin{array}{l}\text { Se analiza la información de todos los nacimientos ocurridos en el año } 2014 \text { y } \\
\text { registrados en } 2014 \text { y } 2015 \text { con desagregación jurisdiccional. }\end{array}$ \\
\hline & & Procedimiento \\
\hline & & $\begin{array}{l}\text { Indirecto; se analizan resultados del Censo } 2010 \text { (hijos nacidos vivos), para } \\
\text { el total del país y sus provincias. Estos datos se comparan con nacimientos } \\
\text { registrados. }\end{array}$ \\
\hline & & Dicha aplicación no es posible para las defunciones infantiles. \\
\hline & & $\begin{array}{l}\text { Directo: Para estimar la cobertura legal y estadística del registro de nacidos } \\
\text { vivos en áreas y establecimientos seleccionados, se utilizó una metodología } \\
\text { consistente en el pareo de fuentes de datos: registros hospitalarios (libros de } \\
\text { partos, resumen del centro obstétrico, etc.), documentación estadística (Informe } \\
\text { Estadístico de Nacido Vivo) y registro civil (acta o libro de inscripción en el } \\
\text { Registro Civil). }\end{array}$ \\
\hline & & $\begin{array}{l}\text { Se trata de un método directo de evaluación de la cobertura del registro, cuyo } \\
\text { aporte más importante consiste en identificar los nacimientos no registrados, } \\
\text { desde el momento mismo de la captación de la información. A partir de la } \\
\text { consideración de listas "completas" —registros hospitalarios_- de todos los } \\
\text { nacimientos acaecidos en los establecimientos bajo estudio, se parea con la } \\
\text { información del registro civil permitiendo conocer los casos de subregistro legal } \\
\text { de nacimientos. }\end{array}$ \\
\hline & & $\begin{array}{l}\text { El pareo entre los registros hospitalarios y los Informes Estadísticos de Nacido } \\
\text { Vivo recibidos en la oficina jurisdiccional de estadísticas vitales permite } \\
\text { cuantificar los casos de subtransmisión estadística. }\end{array}$ \\
\hline
\end{tabular}

\section{Hallazgos Seleccionados}

Método indirecto:

- La omisión de nacidos vivos que surge de comparar la información que brinda el Censo 2010 con los nacidos vivos registrados por el Subsistema de Estadísticas Vitales es del $6 \%$ para el total del país si se toman como referencia los datos publicados por DEIS para el mismo año.

Y se reduce al 3,8 \% si se consideran las inscripciones tardías que se produjeron a lo largo de los años 2011 a 2014. 


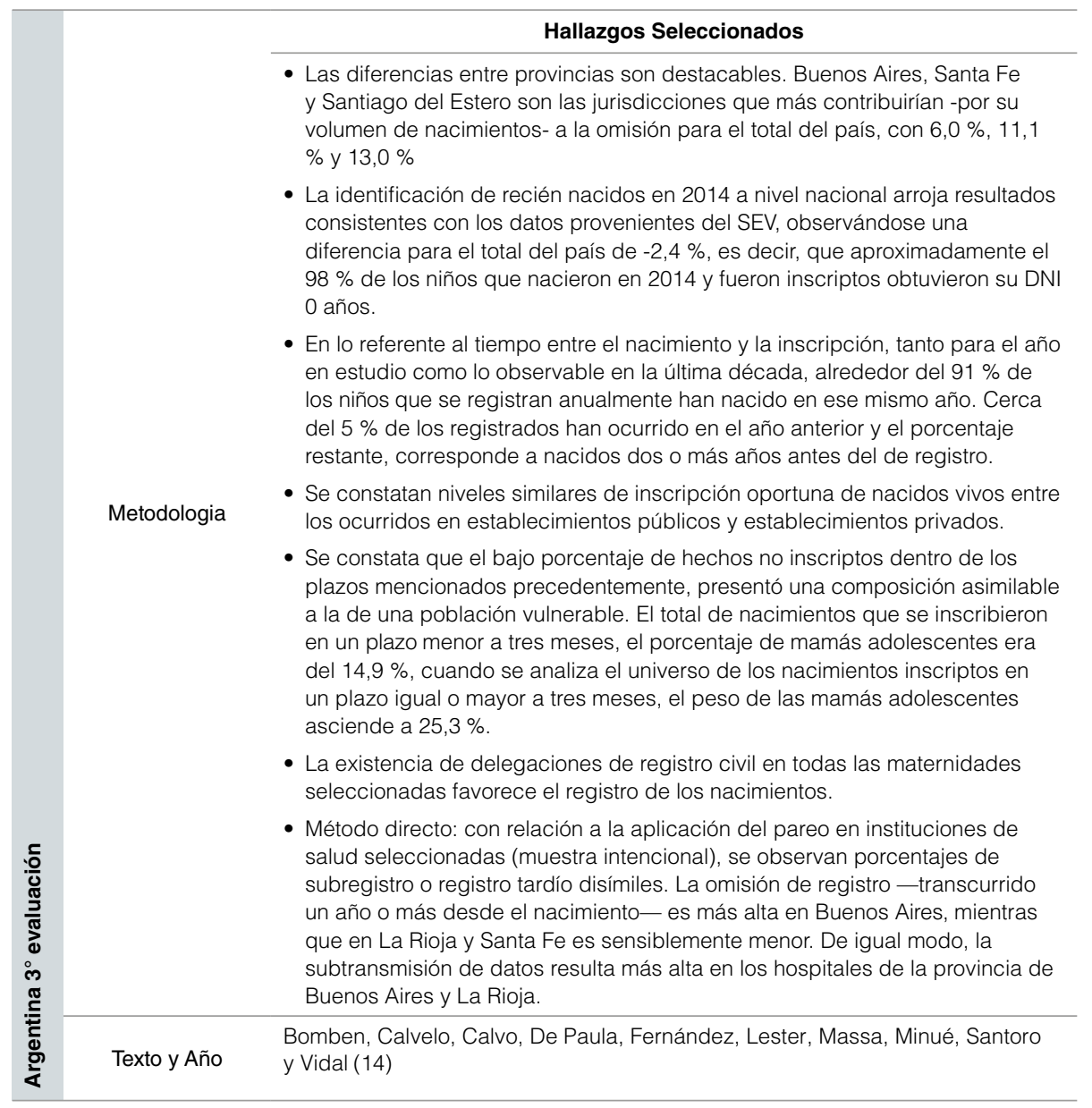

\section{Temporal}

El estudio cuenta con tres etapas, que se describen en apartados diferentes. La primera parte del estudio corresponde al año 2000.

En esta etapa, realizan una búsqueda activa, que está precedida por una evaluación de la adecuación de las estadísticas de nacimiento y de defunción, con base a los resultados obtenidos entre 1996-1998.

\section{Espacial}

Unidades Federativas (UF), y municipios seleccionados de las regiones norte y nordeste

Analizan el registro de defunciones infantiles (incluyendo a las muertes fetales para validar posteriormente el tipo de defunción), con vistas al mejoramiento

Registro Evaluado de la estimación de la mortalidad infantil. La fuente evaluada es el Sistema de Información sobre Mortalidad (SIM, por sus siglas en portugués). Los datos fueron extraídos de DATASUS 


\begin{tabular}{l} 
Técnica/s \\
\hline Se utiliza la Búsqueda activa de defunciones fetales y muertes infantiles. \\
En forma previa, se analiza adecuación de información disponible sobre \\
nacidos vivos y defunciones, hasta el nivel municipal, \\
para identificar situaciones más graves. \\
Con posterioridad a la búsqueda activa, se realiza la corrección de los datos \\
(en apariencia de las UF seleccionadas), \\
para contar con estimación adecuada de las TMI. \\
\hline
\end{tabular}

\section{Procedimiento}

Metodologia

En primer lugar, se evalúa la adecuación de las TMI elaboradas para los años 1996-1998, a través de cinco indicadores de cobertura y calidad:

- Coeficiente general de mortalidad (CGM), estandarizado por edad (< 4 por 1000 indica error).

- Desvío medio del CGM en tres años (< 10 \% indica error).

- Razón entre nacidos vivos informados y estimados (valores bajos indican error).

- Desvío medio relativo de la NV (< $10 \%$ indica error).

- Proporción de defunciones con causas mal definidas (<20\%).

- Con estos indicadores se determina existencia de errores hasta el nivel municipal.

\section{Procedimiento}

Se clasifica a cada municipio según el grado de adecuación (adecuado, no satisface algún criterio, no adecuado).

En segundo lugar, se realiza la búsqueda activa de defunciones del año 2000, teniendo en cuenta 79 municipios con mayores problemas de la región norte (en dos estados) y nordeste (en cuatro estados). A continuación, se seleccionan ocho aglomerados de municipios contiguos en cada UF, para constituir una muestra (no probabilística).

En estos municipios se recolectó, a través de un instrumento estandarizado, información de fuentes oficiales y no oficiales de distinto tipo; por ejemplo, fichas de notificación de los agentes de salud, oficinas de registro civil,

Metodologia cementerios, funerarias, farmacias, establecimientos de salud, y el testimonio de líderes comunales, curanderos y rezadoras.

\section{Hallazgos Seleccionados}

El procedimiento de búsqueda activa permitió identificar subregistro de $66 \%$ de las defunciones infantiles en la muestra analizada. Este porcentaje osciló entre $42 \%$ a $78 \%$ según municipio.

Los procedimientos 1 y 2 permitieron mejorar la estimación de la TMI (15), e identificar los principales problemas del proceso de generación y registro de la declaración de defunciones (16).

Además, el Ministerio de Salud estableció metas de cobertura, acciones de integración entre diferentes subsistemas de información, diseño de proyectos específicos, etc.

Texto y Año Szwarcwald et al., (1), Frias et al., (2)

\section{Temporal}

Se trata de la segunda parte del estudio.

Alcance La búsqueda activa se realiza para hechos ocurridos en el año 2008.

El trabajo de campo se realizó entre 2009 y 2010.

Los resultados fueron utilizados para corregir datos de los años 2000 y 2010. 
Bruno Sebastián Ribotta / Luisa María Salazar Acosta / Carola Leticia Bertone

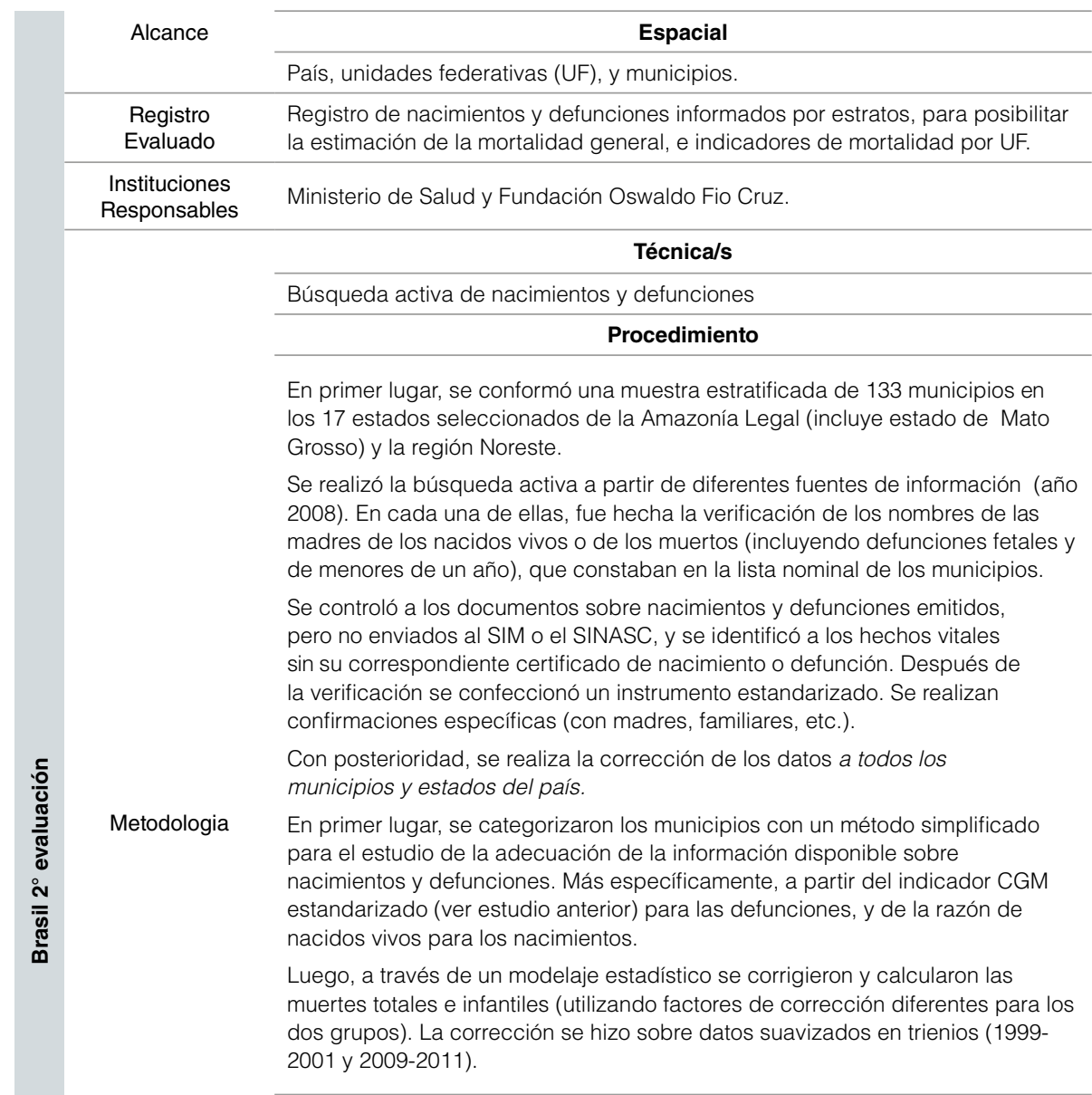

\section{Hallazgos Seleccionados}

Según la generalización de los resultados, la cobertura de las defunciones asciende a $93 \%$ y la de nacimientos a $95 \%$. En las muertes, las UF mejor posicionadas se encuentran al sur y sudeste (prácticamente $100 \%$ ), y las menos favorecidas al norte (80 \% en promedio). En los nacimientos, los porcentajes de cobertura son más homogéneos entre las distintas regiones, siendo de $90 \%$ o más en las distintas UF.

En el 4 \% (230) del total de municipios brasileños, los factores de corrección estimados para las muertes infantiles fueron insuficientes, con registros inferiores al número mínimo esperado de muertes infantiles.

Frias, Szwarcwald, de Souza, da Silva de Almeida, y Cabral Lira (18); Szwarcwald, de Frias, Júnior, da Silva de Almeida, y Neto (25); Victora et al. (26); Almeida y Szwarcwald (27); Szwarcwald, Escalante, Neto, Junior, y

Texto y Año de Publicación Victora (28); França et al. (29); Frias, Szwarcwald, y Lira (30); Szwarcwald et al. (31); Figueiroa, Vanderlei, Frias, Carvalho, y Szwarcwald (32); Almeida y Szwarcwald (33); Frias et al. (34); Szwarcwald, Almeida, y Cortez-Escalante (35) Almeida et al.(36) 
Evaluaciones subnacionales de la cobertura de las estadísticas vitales. Estudios recientes en América Latina

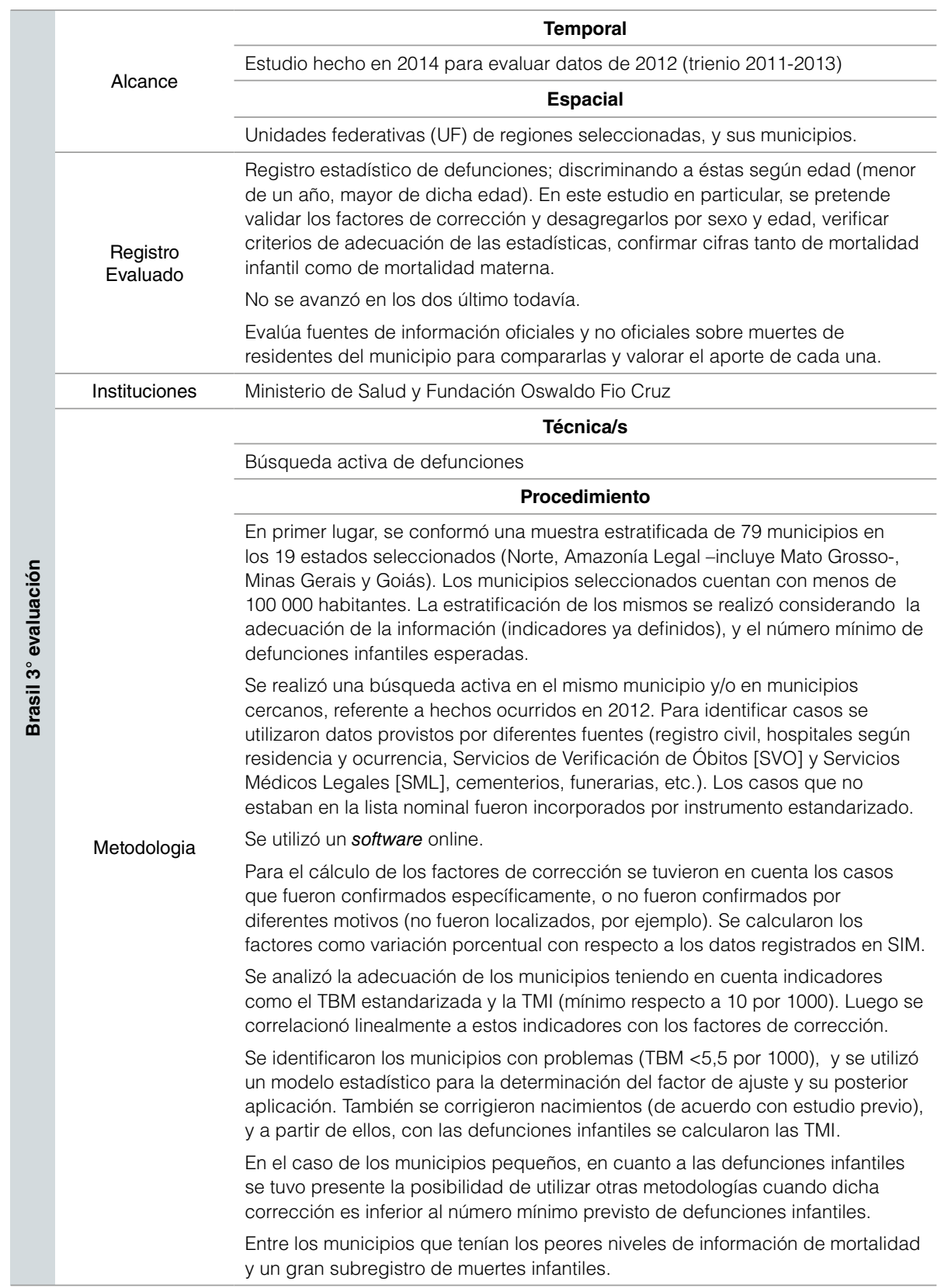




\begin{tabular}{|c|c|c|}
\hline \multirow{10}{*}{ 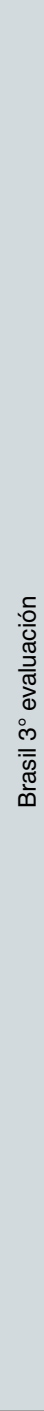 } & \multirow{9}{*}{ Metodologia } & Procedimiento \\
\hline & & $\begin{array}{l}\text { Se investigaron las siguientes fuentes de información: departamentos de } \\
\text { salud estatales y municipales; oficinas de registro; cementerios oficiales y } \\
\text { no oficiales; funerarias; unidades de atención primaria de salud; hospitales } \\
\text { y otras instalaciones de salud (clínicas, unidades de emergencia) del caso } \\
\text { del municipio y municipios vecinos; institutos de medicina forense y servicios } \\
\text { de investigación de la muerte; Registro Unificado de los Programas Sociales } \\
\text { del Gobierno Federal; y las estaciones de policía. Se buscaron agentes } \\
\text { comunitarios de salud y profesionales del equipo de salud familiar, así como } \\
\text { informantes clave como parteras tradicionales y líderes comunitarios. Todas las } \\
\text { fuentes visitadas se registraron en un panel en línea, en el cual se almacenaba } \\
\text { la información de las fuentes y los casos encontrados en el trabajo de campo. }\end{array}$ \\
\hline & & Hallazgos Seleccionados \\
\hline & & $\begin{array}{l}\text { Se observa la persistencia de motivaciones territoriales y culturales en el } \\
\text { subregistro de hechos vitales. Ello es evidente en algunas regiones del norte y } \\
\text { noreste, que aún poseen una cobertura insatisfactoria en el contexto nacional, } \\
\text { por ejemplo; de } 81 \% \text { (Maranhão) y } 85 \% \text { (Amazonas y Pará). Dichos niveles son } \\
\text { más bajos en las defunciones infantiles, al compararlas con las de mayores de } \\
1 \text { año (aproximadamente } 14 \text { puntos porcentuales de diferencia en las regiones } \\
\text { ya mencionadas). }\end{array}$ \\
\hline & & $\begin{array}{l}\text { En solo } 227 \text { municipios no puede estimarse de manera directa a la mortalidad } \\
\text { infantil. Hay alto subregistro y los factores de corrección no son suficientes para } \\
\text { la adecuación de las defunciones infantiles. }\end{array}$ \\
\hline & & $\begin{array}{l}\text { Encontramos } 2265 \text { muertes que no fueron informadas al Sistema de Información } \\
\text { de Mortalidad. De ellos, el } 49,3 \text { \% se encontraron en fuentes no oficiales, } \\
\text { cementerios y funerarias. En algunos municipios rurales, se encontraron } \\
\text { condiciones de entierro precarias en los cementerios en el medio del bosque y } \\
\text { sin registro de los fallecidos. En cuanto a la adecuación de la información sobre } \\
\text { las muertes infantiles, } 16(20,3 \%) \text { eran inadecuadas. }\end{array}$ \\
\hline & & $\begin{array}{l}\text { Los factores de corrección se asociaron inversamente con el nivel de } \\
\text { adecuación de la información de mortalidad. }\end{array}$ \\
\hline & & $\begin{array}{l}\text { La Compañía de Tecnología e Información de Seguridad Social (SISOBI / } \\
\text { DATAPREV) podría ser una fuente alternativa interesante para el uso rutinario y } \\
\text { la captura de muertes registradas en las oficinas de registro y no informadas a } \\
\text { la SIM. }\end{array}$ \\
\hline & & $\begin{array}{l}\text { Hay una ausencia parcial de datos debido a problemas que se multiplican } \\
\text { geográficamente, zonas socioeconómicas desfavorecidas con dificultades } \\
\text { de acceso a la asistencia sanitaria y que son diversificado por los aspectos } \\
\text { culturales de las comunidades locales }\end{array}$ \\
\hline & $\begin{array}{l}\text { Texto y Año de } \\
\text { Publicación }\end{array}$ & Szwarcwald, Almeida, y Cortez Escalante, (35) Almeida, W.S. et al. (36) \\
\hline \multirow{6}{*}{$\begin{array}{l}\text { 음 } \\
\text { 흥 }\end{array}$} & \multirow{4}{*}{ Alcance } & Temporal \\
\hline & & 2002 \\
\hline & & Espacial \\
\hline & & Nivel nacional y departamental \\
\hline & $\begin{array}{l}\text { Registro } \\
\text { Evaluado }\end{array}$ & $\begin{array}{l}\text { Se realiza la evaluación de la cobertura y oportunidad del registro de nacimiento, } \\
\text { y de cobertura del registro de defunción (considerando específicamente muerte } \\
\text { materna y perinatal; fetal tardía y neonatal precoz). }\end{array}$ \\
\hline & $\begin{array}{l}\text { Instituciones } \\
\text { Responsables }\end{array}$ & DANE y el Ministerio de la Protección Social, con apoyo financiero de UNFPA. \\
\hline
\end{tabular}




\begin{tabular}{|c|c|c|}
\hline \multirow{12}{*}{$\frac{\frac{\pi}{0}}{\frac{\text { ह }}{0}}$} & \multirow{12}{*}{ Metodologia } & Técnica/s \\
\hline & & $\begin{array}{l}\text { La principal técnica que utilizan es directa, de pareo de distintas fuentes de } \\
\text { datos, complementada encuestas y autopsia verbal. También se recurre a } \\
\text { comparación de series estadísticas. }\end{array}$ \\
\hline & & Procedimiento \\
\hline & & $\begin{array}{l}\text { En el análisis de la cobertura se utilizaron numerosas fuentes de datos: historias } \\
\text { clínicas, autopsias verbales, encuestas a parteras, seriales de hechos vitales en } \\
\text { instituciones de salud, registro civil, medicina legal y registros de inhumación, etc.). }\end{array}$ \\
\hline & & $\begin{array}{l}\text { En cuanto a la cobertura de los nacimientos, se busca identificar los faltantes a } \\
\text { partir de certificados en instituciones de salud (partos institucionales) o notarías }\end{array}$ \\
\hline & & $\begin{array}{l}\text { - registradurías (partos no institucionales), y el DANE, a través del número serial } \\
\text { de los certificados. Se complementa la evaluación de los partos no institucionales } \\
\text { consultando a parteras u otros agentes que hayan intervenido en los mismos. }\end{array}$ \\
\hline & & $\begin{array}{l}\text { Con el mismo fin se elaboran certificados estándar basados en historias clínicas de } \\
\text { mujeres en edad fértil que tenían consignado un nacimiento además de la defunción, y } \\
\text { autopsias verbales (de mujeres que mencionaron haber tenido hijos nacidos vivos en el } \\
\text { año de referencia, y de muertes perinatales en los que se identificaron nacidos vivos). }\end{array}$ \\
\hline & & $\begin{array}{l}\text { Para el estudio de la cobertura del registro de muertes, se comparan números } \\
\text { seriales de certificados de instituciones de salud y notarías o registradurías, con los } \\
\text { equivalentes en el DANE, y con el mismo fin comparativo se elaboran certificados a } \\
\text { partir de la historia clínica (institucional) autopsias verbales (no institucional). }\end{array}$ \\
\hline & & $\begin{array}{l}\text { La metodología tiene adaptaciones específicas para defunciones perinatales. En análisis } \\
\text { de la exactitud de la información sobre ambos hechos vitales se realiza comparando los } \\
\text { certificados del DANE, con los realizados especialmente (a partir de historias clínicas, } \\
\text { autopsias verbales, encuestas y entrevistas). Para la confección de dichos certificados } \\
\text { existen nueve formatos diferentes (según el hecho vital y característica específica: mujer } \\
\text { en edad fértil, muerte neonatal, etc.) y un cuestionario de encuesta (parteras). }\end{array}$ \\
\hline & & $\begin{array}{l}\text { El procedimiento descripto se aplica a una muestra probabilística estratificada, } \\
\text { de } 3500 \text { certificados que involucra a } 1118 \text { municipios, con representatividad } \\
\text { para } 22 \text { departamentos, dos grupos para los antiguos territorios nacionales, uno } \\
\text { para San Andrés y Providencia y uno para Bogotá D.C.; }\end{array}$ \\
\hline & & $\begin{array}{l}\text { - En nacimientos se considera un } 2 \text { por } 1000 \text { de cada municipio con excepción } \\
\text { de: Bogotá DC (1 por 1000), municipios pequeños (todos los nacimientos si } \\
\text { hay } 2 \text { o menos por año); }\end{array}$ \\
\hline & & - 326 parteras (nacimientos no institucionales). \\
\hline
\end{tabular}

\begin{tabular}{|c|c|c|}
\hline \multirow{7}{*}{$\begin{array}{l}\frac{\pi}{0} \\
\frac{0}{0} \\
\frac{0}{0}\end{array}$} & & Hallazgos Seleccionados \\
\hline & \multirow{5}{*}{ Metodologia } & $\begin{array}{l}\text { A nivel nacional, está registrado el } 86 \% \text { de los nacimientos. A nivel departamental, } \\
\text { los contrastes más importantes se observan entre Bogotá ( } 95 \% \text { ), y Chocó (57 \%). } \\
\text { La cobertura de defunciones es de } 90 \% \text {. Nuevamente, las diferencias más grandes } \\
\text { se encuentran entre Bogotá (95\%) y Chocó ( } 64 \% \text { ). Los niveles de cobertura } \\
\text { se explicarían por el nivel de desarrollo de los departamentos y por la agencia } \\
\text { departamental del DANE que cubre el registro. }\end{array}$ \\
\hline & & $\begin{array}{l}\text { En las defunciones perinatales la cobertura es menor; } 73 \% \text { para el total, que } \\
\text { puede desagregarse en } 83 \% \text { de neonatales precoces y } 67 \% \text { de fetales. }\end{array}$ \\
\hline & & $\begin{array}{l}\text { Existen departamentos donde el } 50 \% \text { de estas defunciones no se registran } \\
\text { (mayoría de los que conforman costa Atlántica). }\end{array}$ \\
\hline & & $\begin{array}{l}\text { Chocó posee el porcentaje más alto de subregistro ( } 24 \% \text { ), al igual que la } \\
\text { mayoríade los departamentos de la costa atlántica. }\end{array}$ \\
\hline & & $\begin{array}{l}\text { Se realiza un análisis de la coherencia de los resultados con pautas ya conocidas, y } \\
\text { en el caso de los nacimientos, con resultados de la ENDS 2005. También se comparan } \\
\text { las tasas con datos registrados y corregidos por cobertura. }\end{array}$ \\
\hline & $\begin{array}{l}\text { Texto y Año de } \\
\text { Publicación }\end{array}$ & DANE y Dirección de Censos y Demografía (37) \\
\hline
\end{tabular}




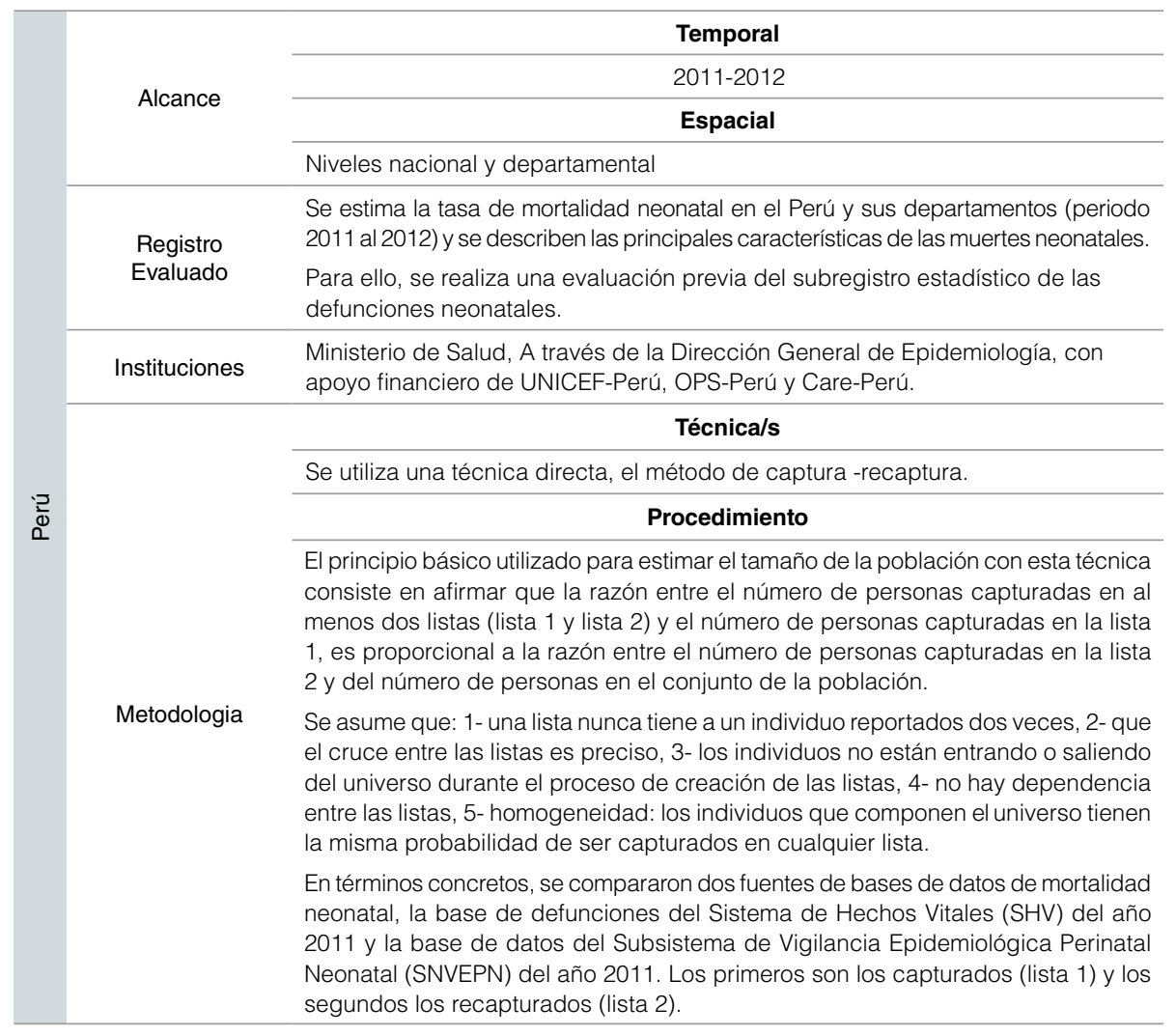

\section{Procedimiento}

\begin{tabular}{|c|c|c|}
\hline \multirow{10}{*}{ 깅 } & \multirow{9}{*}{ Metodologia } & Procedimiento \\
\hline & & $\begin{array}{l}\text { Se parearon los registros en ambas bases de datos, por apellidos, nombres, edad } \\
\text { y procedencia, y diagnóstico. Considerando las categorías "Coincide", }\end{array}$ \\
\hline & & $\begin{array}{l}\text { "Falta en Base 2", "Falta en base 1", se construyó una tabla dinámica para } \\
\text { desarrollar reportes y estimar el número de muertes neonatales, calculando el } \\
\text { porcentaje de subregistro por cada base. }\end{array}$ \\
\hline & & $\begin{array}{l}\text { Los dos primeros supuestos de la técnica fueron controlados durante el proceso } \\
\text { de organización de los datos y la fase de cruce de listas; se hicieron pruebas de } \\
\text { consistencia tanto durante el proceso de depuración de registros duplicados (dentro de } \\
\text { cada lista) como a lo largo de la fase de cruce de listas(a través de las diferentes listas). }\end{array}$ \\
\hline & & Hallazgos Seleccionados \\
\hline & & $\begin{array}{l}\text { EI SNVEPN tiene un subregistro en la captación de muertes neonatales de 52,9\% (IC: } \\
51,7 \%-54,1 \% \text { ), frente a un mayor subregistro del SHV } 66,6 \% \text { (IC: } 65,1 \%-68,1 \% \text { ). }\end{array}$ \\
\hline & & $\begin{array}{l}\text { Si se unen las bases de datos, el subregistro de mortalidad neonatal en el Perú } \\
\text { para el año } 2011 \text { se estima en } 24,6 \% \text { (IC: } 24,1 \%-25,3 \%) \text {. }\end{array}$ \\
\hline & & $\begin{array}{l}\text { La región con mayor subregistro es la selva y la zona rural del país. Los quintiles } \\
\text { de mayor pobreza concentran mayor subregistro, sobre todo en SHV. A mayor } \\
\text { número de días de sobrevida, mayor es el subregistro. }\end{array}$ \\
\hline & & $\begin{array}{l}\text { No se realiza consistencia específica. Si se analizó la exhaustividad de las bases. } \\
\text { Al verificar que ninguna de ellas cumple con el requisito ( } 80 \text { \% o más), el análisis } \\
\text { de la mortalidad se realizó considerando la combinación de ambas. }\end{array}$ \\
\hline & Texto y Año & $\begin{array}{l}\text { Avila Vargas Machuca, Tavera Salazar, y Carrasco Gamarra (38); Ávila, } \\
\text { Tavera, y Carrasco(35) }\end{array}$ \\
\hline
\end{tabular}




\section{Notas}

1 Primera Conferencia Regional Latinoamericana sobre el Derecho a la Identidad y el Registro Universal de Nacimiento (Paraguay, 2007; http://www.unicef.org/lac/overview_9654.htm) y Segunda Conferencia Regional Sobre el Derecho a la Identidad, Panamá, 2011; http:// www.unicef.org/lac/overview_21634.htm). También constituyen un ejemplo de este accionar el programa para el registro legal de nacimientos impulsado por el Banco Interamericano de Desarrollo.

2 Pan American Health Organization. Regional Plan of Action for Strengthening of Vital and Health Statistics. 48th Directing Council of PAHO, 60th Session of the Regional Committee of WHO for the Americas; Washington DC, USA; Sept 29-Oct 3, 2008. http://www1.paho. org/english/gov/cd/cd48.r6-e.pdf

3 Relacsis es una comunidad académica y de práctica que fomenta la cooperación entre profesionales del área, la capacitación de recursos humanos en los países de la región, la generación de acciones en común y la compilación y difusión de buenas prácticas para el fortalecimiento de los sistemas rutinarios de información en salud (http://www.paho.org/ relacsis/).

4 La medición del subregistro fue aproximada a partir de la diferencia relativa entre los datos registrados y los provistos por proyecciones de población.

5 Los métodos indirectos son evaluaciones basadas en los resultados del sistema de información en sí mismo (es decir, que comparan datos cuantitativos obtenidos con cálculos de mayor a menor complejidad, a partir de la misma fuente que se evalúa o de otras fuentes de datos que proporcionan información similar o emparentada a la que se desea evaluar, aunque esta sea incompleta o en alguna medida incorrecta). Los principales tipos de métodos indirectos para la evaluación de la cobertura de nacimientos y defunciones son la comparación de tendencias, el análisis de las inscripciones tardías, la comparación con datos censales (por ejemplo, con la población menor de un año), el uso de preguntas sobre registro en encuestas por muestreo, la comparación con poblaciones similares o en periodos anteriores y las técnicas de estimación con datos incompletos, o técnicas "indirectas", entre otros (4: 139).

6 Algunas excepciones están representadas, por ejemplo, en evaluaciones basadas en metodologías indirectas como la realizada por Lima y Queiroz (10), que analiza la cobertura del registro de nacimientos y defunciones en las unidades federativas de Brasil, o por Unicef e INEGI (11), que estudia la oportunidad del registro de hechos vitales en los estados y municipios de México. En estos países, la aplicación de dichas metodologías resulta factible principalmente en el elevado tamaño poblacional (ambos constituyen los más habitados de la región).

7 En este trabajo, la definición y caracterización de los métodos directos para el estudio de la cobertura se basa principalmente en las propuestas de Naciones Unidas (3, p. 94; 4, p. 136) y Moultrie et al. (12, p. 6).

8 Naciones Unidas (4, p. 140). 
9 Se consideran práctica que también utilizan metodologías indirectas, pero de forma complementaria o accesoria.

10 Se excluyen las prácticas referidas con exclusividad a la evaluación directa del registro de muertes maternas, dado que exceden los objetivos de nuestra investigación actual.

11 La fecha de referencia intenta reflejar los esfuerzos para el mejoramiento de los sistemas de información sanitarios, que se renuevan notoriamente a partir de la Cumbre del Milenio, con miras al seguimiento de los indicadores de salud relacionados con los ODM 4, 5 y 6. Véase al respecto "La salud y los Objetivos de Desarrollo del Milenio" (13).

12 Véase http://www.paho.org/relacsis/index.php/areas-de-trabajo/gt11-cobertura-y-calidad/ item/649-foro-cobertura-de-nacimientos-y-defunciones

13 Véase http://www.cepal.org/es/enlaces-los-institutos-nacionales-de-estadistica-en-america-latina-y-el-caribe

14 Véase http://www.paho.org/hq/index.php?option=com_weblinks\&view=category\&i$\mathrm{d}=28 \% 3$ Aministries-health\&Item id=941\&lang $=$ es

15 Nos referimos a investigaciones realizadas en Brasil $(10,14,15)$, Colombia (16) y México $(11,17)$.

16 Las DAM corresponden a las provincias en Argentina, los departamentos en Colombia y Perú, y los Estados o unidades federativas (UF) en Brasil. En este último país, las UF se agrupan en regiones.

17 En la práctica del año 2000 se trata de las regiones del norte y nordeste. En el estudio del año 2008 se incorpora el estado de Mato Grosso (incorporando así la llamada Amazonía Legal). Finalmente, en el trabajo de 2012 se incluye también a Minas Gerais y Goiás. Cabe destacar que en la segunda práctica, no se consideran todas las UF de las regiones elegidas, sino las 17 con mayores problemáticas.

18 Solo se cuantifica la cobertura de las muertes infantiles. No obstante, la búsqueda incluye a las defunciones fetales con el objetivo de validar el tipo de muerte.

19 Cabe destacar que, en Argentina, dicha evaluación corresponde más a las causas del problema que a la cobertura de este, por los recortes espaciales antes mencionados (determinadas provincias y dentro de estas, ciertas instituciones de salud). En esta práctica sí se proporciona una estimación de la cobertura de los nacimientos, pero a partir de una indagación de tipo indirecta.

20 Menores de un año y mayores de esa edad.

21 Defunciones neonatales y maternas.

22 En Brasil, Colombia y Perú, los sistemas de estadísticas vitales se apoyan en primer instancia en el sector salud (40-42).

23 En demografía este principio se utiliza muy corrientemente en la técnica del "sistema de doble registro", ya que compara los resultados de una encuesta con el registro civil, aplicando la fórmula estadística de Sekar y Deming (44). 
24 La metodología descrita no debería confundirse con un procedimiento de carácter rutinario aplicado en México. En este país, la Secretaría de Salud realiza a nivel estatal, y de manera sistemática, una "búsqueda intencionada de muertes". El objetivo es ubicar defunciones no registradas, especialmente de menores de cinco años. La base de datos construida con información de las instituciones de salud se confronta con la del Subsistema Estadístico y Epidemiológico de las Defunciones (SEED), y si existen diferencias se solicita y analiza la tercera copia del certificado de defunción que se implementa en dicho país (46).

25 En este caso, la comprobación indirecta corresponde más bien a una validación de lo obtenido en la estimación directa, requisito previsto entre los principios de la evaluación de los datos. Es decir, de la coherencia o consistencia de un resultado, con el obtenido mediante otra estimación, basada en técnicas e información independiente (47).

26 Como fuera anticipado, la metodología indirecta consiste en contrastar el dato registrado con el obtenido en el censo de población y vivienda de 2001. En dicho relevamiento, no resulta posible analizar a las defunciones infantiles porque los datos sobre hijos sobrevivientes no cumplen con los estándares de calidad recomendados.

27 Resulta de comparar el dato registrado según publica el Ministerio de Salud, con nacimientos estimados en proyecciones de población (48).

28 Comparación del dato registrado según publica el DANE, con nacimientos estimados en proyecciones de población (49).

29 Las cifras son de las UF Distrito Federal y Maranhão.

30 éase Celade (50). 30

31 Una iniciativa que podría replicarse a casi diez años de su realización es el informe regional sobre la "Situación de las estadísticas vitales, de morbilidad y de recursos y servicios en salud de los países de las Américas”, que elaboró la OPS/OMS a partir de un diagnóstico realizado en el año 2005, en el marco de las tareas preparatorias para la creación del PEVS (8). 